\title{
Soil heterogeneity and preferential paths for gas migration
}

\author{
C.H. Delahaye ${ }^{\mathrm{a}, \mathrm{b}, *}$, E.E. Alonso ${ }^{\mathrm{a}}$ \\ ${ }^{a}$ Geotechnical Engineering Department, Technical University of Catalonia (DIT-UPC), Barcelona, Spain \\ ${ }^{\mathrm{b}}$ Institute of Mining Research, National University of San Juan (IIM-UNSJ), San Juan, Argentina
}

Received 24 July 2000; accepted 31 July 2001

\begin{abstract}
Preferential paths are often reported in connection with gas breakthrough experiments. These preferential paths may be explained, in a deformable soil skeleton, by the action of pressurised gas along a natural path. A natural path is defined in a spatially heterogeneous material by a set of connected spots of higher gas permeability. Along this path the soil will experience a marked desaturation and gas permeability will increase fast. The paper describes a procedure to simulate these effects. Soil heterogeneity is described by means of random fields, which describe the key soil properties for gas migration. Since actual data concerning soil variability at a small (sample) scale is very limited, a number of computer models of increased complexity have been developed. They maintain, however, a close relationship with the known macroscopic data of the average (homogeneous) sample. The effect of some hypothesis concerning the stochastic structure of soil variability and the role of the hydromechanical coupling has also been explored. Once the heterogeneous soil model is defined, a general purpose code for Thermo-HydroMechanical analysis is subsequently used to perform the analysis. The soil is initially saturated and a non-wetting phase (gas) is forced through the saturated mass either at constant pressure or at a constant flow rate. The results of the different analyses are compared in order to advance in the basic understanding of gas migration through clay barriers. Finally, a discussion of the relevance of soil heterogeneity, on the basis of the analysis performed, is presented. (C) 2002 Elsevier Science B.V. All rights reserved.
\end{abstract}

Keywords: Soil heterogeneity; Preferential paths; Gas migration

\section{Introduction}

In a previous paper (Delahaye and Alonso, 1997), gas migration through the soil was analyzed by means of a continuum multiphase flow approach. A fully coupled Thermo-Hydro-Mechanical code (CODEBRIGHT, Olivella et al., 1994, 1996) was used in an attempt to simulate some gas migration experiments performed within the framework of MEGAS project

\footnotetext{
* Corresponding author. Geotechnical Engineering Department, Technical University of Catalonia (DIT-UPC), 08034 Barcelona, Spain.
}

(Volckaert et al., 1995). The most significant soil properties of Boom Clay (water retention curve, water and gas relative permeabilities) were taken from actual measurements reported in Volckaert et al. (1995) and Ortiz et al. (1997). A sensitivity analysis was also performed by varying the water retention characteristics of the soil and, specially, its mechanical properties. It was found that the profile of degree of saturation along the sample, which was determined once the experiments were finished, could be matched quite precisely by the model. Other computed results were, however, very far from actual measurements (time to breakthrough, gas flow rate, variation of gas flow rate 
Table 1

Constitutive equations and equilibrium restrictions

\begin{tabular}{lll}
\hline Equation & Variable name & Variable \\
\hline $\begin{array}{l}\text { Constitutive equations } \\
\text { Darcy's law }\end{array}$ & $\begin{array}{l}\text { liquid and gas } \\
\text { advective flux } \\
\text { vapour and dry gas } \\
\text { non-advective fluxes } \\
\text { Fick's law }\end{array}$ & $q_{1}, q_{\mathrm{g}}$ \\
$\begin{array}{l}\text { Conductive heat flux } \\
\text { Fourier's law }\end{array}$ & $i_{\mathrm{c}}{ }^{\mathrm{a}}$ \\
Retention curve & $\begin{array}{l}\text { Liquid phase degree } \\
\text { of saturation }\end{array}$ & $S_{1}, S_{\mathrm{g}}$ \\
Mechanical & Stress tensor & $\sigma$ \\
constitutive model & $\begin{array}{l}\text { Liquid density } \\
\text { Ghase density } \\
\text { Gases law }\end{array}$ & $\rho_{1}$ \\
Equilibrium restrictions & $\rho_{\mathrm{g}}$ \\
Henry's law & $\begin{array}{l}\text { Dry gas dissolved } \\
\text { mass fraction }\end{array}$ & $\omega_{1}^{\mathrm{a}}$ \\
Psychrometric law & Vapour mass fraction & $\omega_{\mathrm{g}}{ }^{\mathrm{w}}$ \\
\hline
\end{tabular}

with time). It was found that the mechanical properties of the sample had an important effect on the computed variation of gas pressure at one of the sample bounda- ries where the flow rate of gas was imposed. When soil stiffness was reduced computed results became closer to actual measurements. This effect could be explained as follows: for low stiffness of the soil skeleton, an increase in gas pressure results in substantially higher porosities than the initial value. Soil intrinsic permeability changes fast with porosity and therefore gas flow rates increase rapidly, approaching actual measurements. However, in order to reproduce test results, soil stiffness had to be reduced to unrealistic values.

It was therefore decided to explore other alternatives, namely those that lead to preferential pathways. Visual examination of samples, once the gas migration experiments mentioned before were performed, suggested that gas flow was concentrated on some preferential paths. Two mechanisms have been suggested: (a) Natural pre-existing soil heterogeneity leads to a preferential path for gas migration. Along this path, the soil will experience a marked desaturation and gas permeability will increase fast. (b) A gas pressureinduced fracture path opens, in a process very similar to the usual hydraulic fracture mechanism, a phenom-

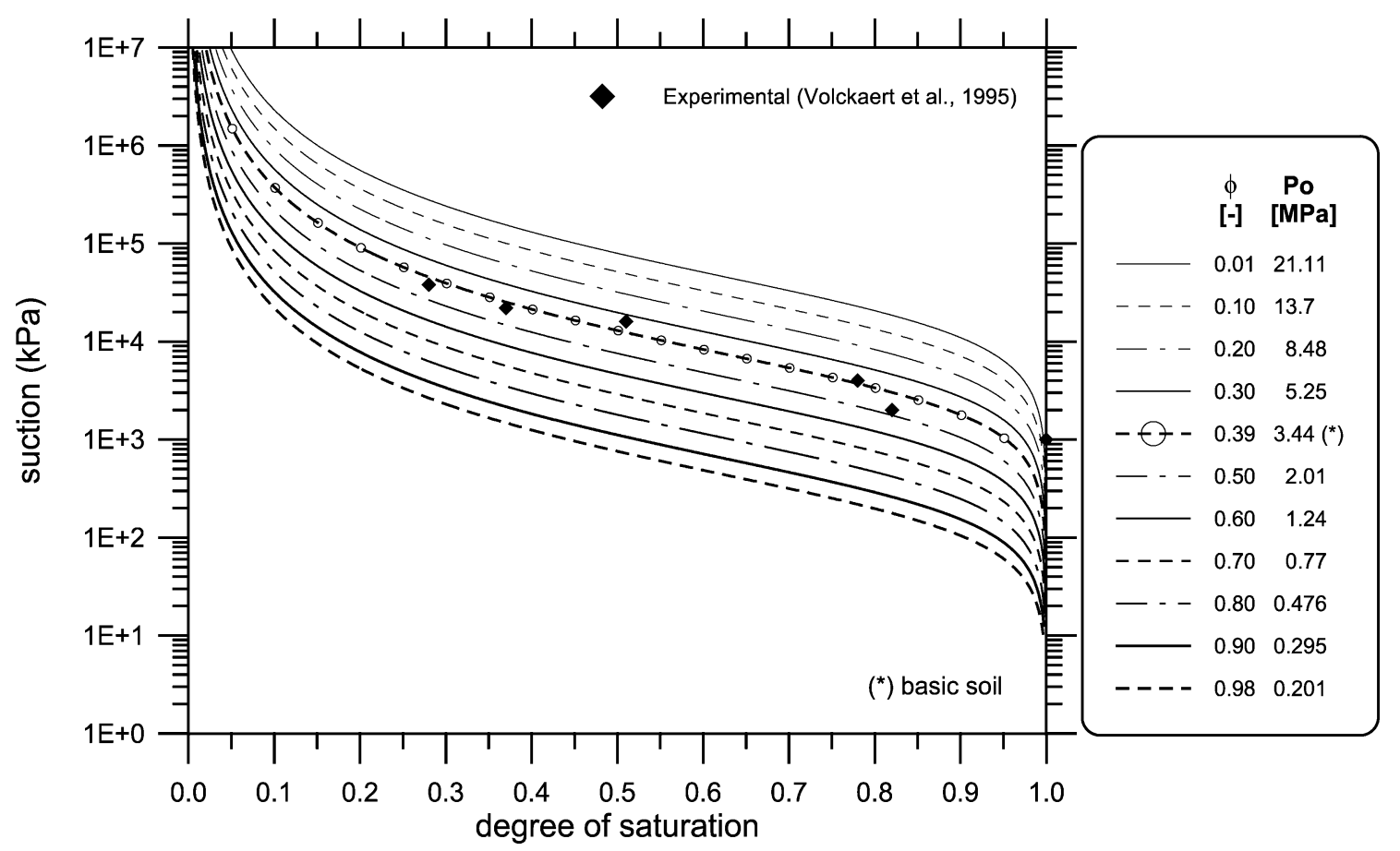

Fig. 1. Water retention curves for the homogeneous and heterogeneous cases. 


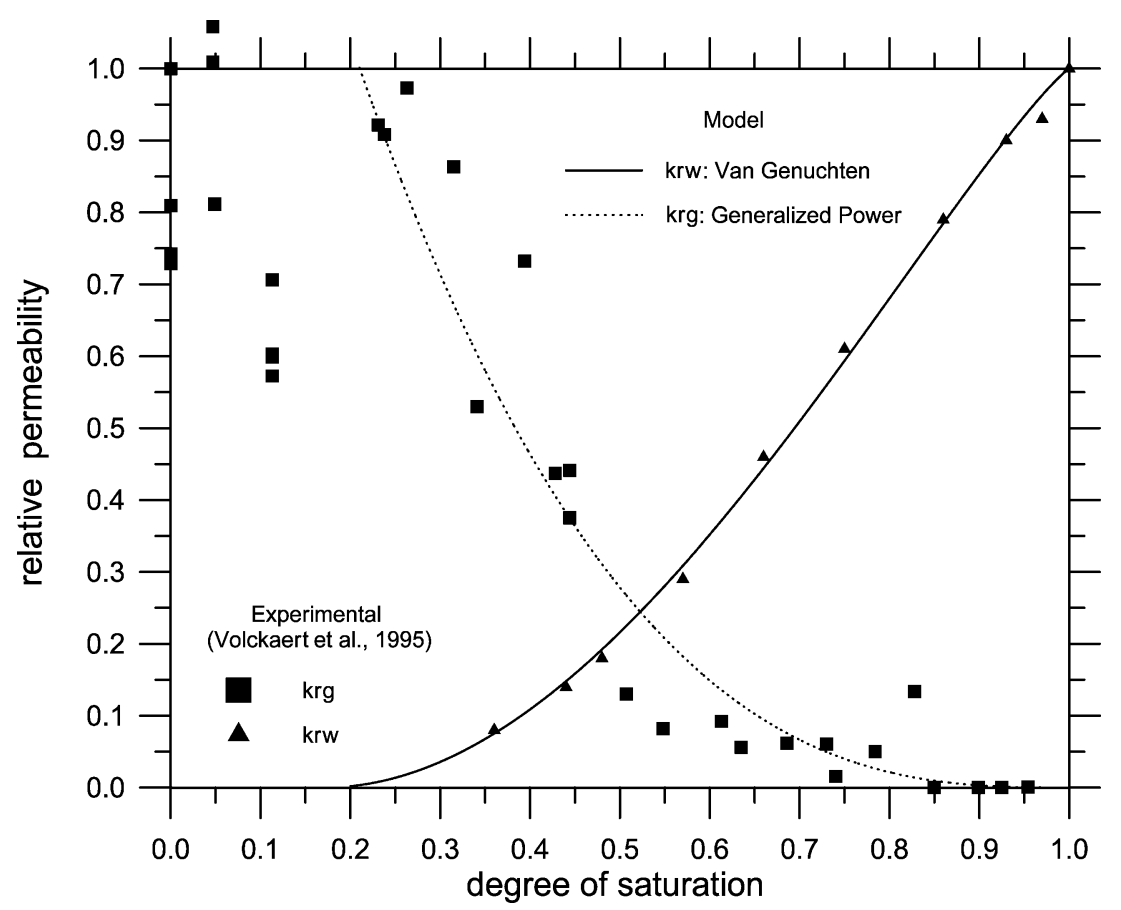

Fig. 2. Relative permeability data of Boom clay specimens and relationships adopted for the homogeneous and heterogeneous cases.

enon which has been extensively analysed in the field of earthdam engineering (Alonso, 1997).

It has been shown (Aylmore, 1993; Brun, 1989; Conciani et al., 1995) that soil heterogeneity leads to a distinct fingering phenomena when a wetting front advances through an unsaturated soil. In our case, the process is not the same: the soil is initially saturated and a non-wetting phase (gas) is forced through the saturated mass either at constant pressure or at a constant flow rate. But one may suspect that some similarities exist between both phenomena, in the sense that high permeability paths within the soil mass control the advance of either a wetting or a desaturation front.

The two mechanisms outlined above are probably related. In fact, a gas pressure-induced fracture is likely to initiate at a point where the gas pressure is maximum (lowest "net" stress). The distribution of gas pressure, on the other hand, is controlled by local variations in permeability and water retention properties. This paper deals with the first mechanism mentioned. It describes the procedure to generate random fields for soil properties that control the flow of gas through soils. A few different cases have been considered to explore the nature of the phenomena involved.
Since actual data concerning soil variability at a small (sample) scale is very limited, a number of computer models of increased complexity have been developed. They maintain, however, a close relationship with the known macroscopic data of the average (homogeneous) sample, as explained below. The effect of some hypothesis concerning the stochastic structure of soil

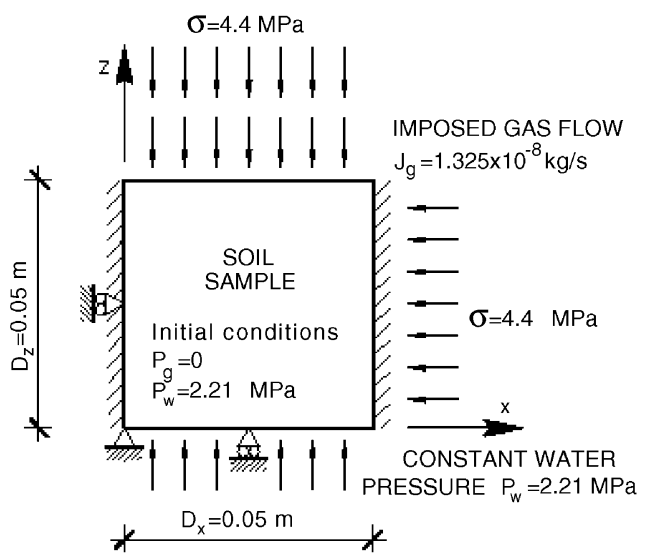

Fig. 3. Definition of solved case. 
Table 2

Definition of cases

\begin{tabular}{|c|c|c|c|c|c|c|}
\hline \multirow[t]{3}{*}{ Soil properties } & \multicolumn{6}{|l|}{ Case } \\
\hline & \multirow{2}{*}{$\begin{array}{l}\text { Homogeneous } \\
\text { Case } 1\end{array}$} & \multicolumn{5}{|c|}{ Randomly heterogeneous } \\
\hline & & Case 2 & Case 3 & Case 4 & Case 5 & Case 6 \\
\hline $\begin{array}{l}\text { Intrinsic permeability } \\
\quad \operatorname{Var}\left(\log _{10} K\right)^{\mathrm{a}}\end{array}$ & 0 & 2 & 2 & 2 & 2 & 2 \\
\hline Porosity, $n$ & 0.39 & 0.39 & $\begin{array}{l}\text { Associated with } K \\
\text { (cubic law) }\end{array}$ & $\begin{array}{l}\text { Associated with } K \\
\text { (cubic law) }^{\mathrm{b}}\end{array}$ & $\begin{array}{l}\text { Associated with } K \\
\text { (cubic law) }^{\text {b }}\end{array}$ & $\begin{array}{l}\text { Associated with } K \\
\text { (cubic law) }^{\mathrm{b}}\end{array}$ \\
\hline Retention curve & Constant (Basic) ${ }^{\mathrm{c}}$ & Basic $^{c}$ & Basic $^{c}$ & $\begin{array}{l}\text { Changing with } n, \\
P_{0}(n)^{\mathrm{d}}\end{array}$ & Basic & $\begin{array}{l}\text { Changing with } n \text {, } \\
P_{0}(n)^{\mathrm{d}}\end{array}$ \\
\hline $\begin{array}{l}\text { Relative permeability } \\
\text { (gas and liquid) }\end{array}$ & Constant $(\text { Basic })^{\mathrm{e}}$ & Basic $^{e}$ & Basic $^{\mathrm{e}}$ & Basic & Basic & Basic \\
\hline $\begin{array}{l}\text { Stress-strain law } \\
E(\mathrm{MPa}) \\
v\end{array}$ & Rigid & Rigid & Rigid & Rigid & $\begin{array}{l}\text { Linear elastic } \\
75 \\
0.333\end{array}$ & Elastoplastic $(\mathrm{BBM})^{\mathrm{f}}$ \\
\hline
\end{tabular}

${ }^{\text {a }} K$ : Intrinsic permeability. Average value for the homogeneous case $K_{0}=4.6 \times 10^{-19} \mathrm{~m}^{2}$. See text for a discussion of the value of $K_{0}$ for the heterogeneous samples.

${ }^{\mathrm{b}} n$ is backcalculated from Kozeny's cubic law. $K=K_{0} n^{3}\left(1-n_{0}\right)^{2} /(1-n)^{2} n_{0}^{3} \quad\left(K_{0}=4.6 \times 10^{-19} \mathrm{~m}^{2}, n_{0}=0.39\right)$.

c See Fig. 2.1 (for $n=0.39, P_{0}=3.443 \mathrm{MPa}$ ).

d The range of retention curves used is indicated in Fig. 2.1. These curves have common parameters except for the $P_{0}$ value, which varies with $n$ as indicated in Fig. 2.8.

${ }^{\mathrm{e}}$ Models for krg and krw are shown in Fig. 2.2.

${ }^{f}$ BBM: Barcelona Basic Model. See text.

variability and the role of the hydro-mechanical coupling has also been examined. The computed results are reported in terms of spatial distributions and time histories of some flow properties (flow rate, pressures).

Once the heterogeneous soil model is defined, the computer model is subsequently used to perform the analysis. Therefore, a basic consistent framework for hydro-mechanical interactions is maintained. The results of the different analyses performed will be compared in order to advance in the basic understanding of gas migration. In a final chapter, a discussion of the relevance of soil heterogeneity, on the basis of the analysis performed, will be presented.

\section{Theoretical bases}

The hydro-mechanical analyses performed have taken into account the following phenomena:

Gas flow:

- Gas phase (Darcy's law).

- Dissolution of gas in the liquid phase (Henry's law).
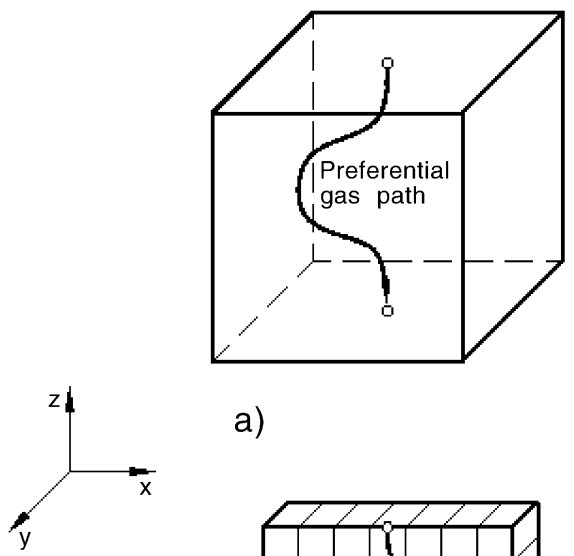

a)

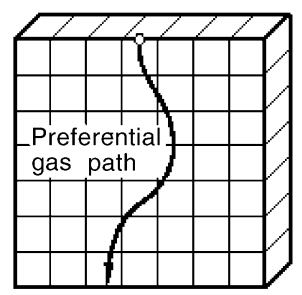

b)

Fig. 4. Preferential path in an heterogeneous sample. (a) Threedimensional condition; (b) two-dimensional condition. 


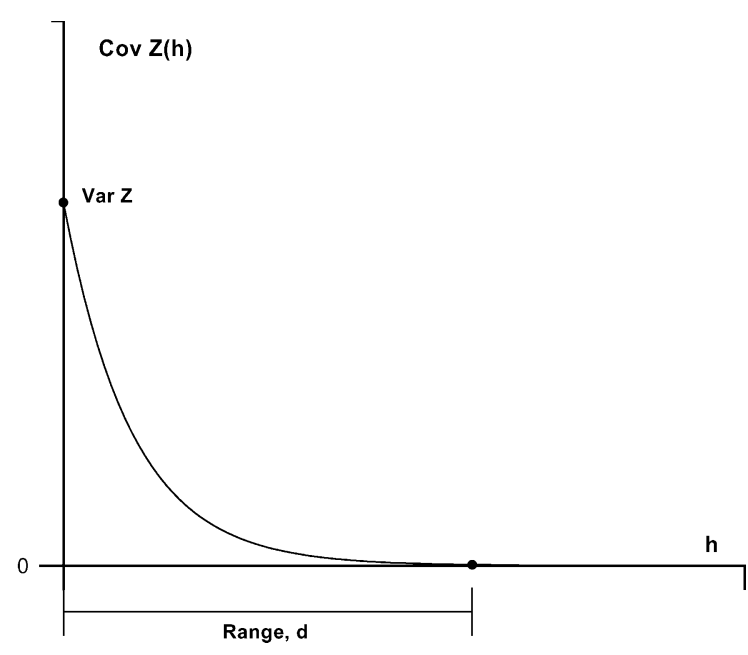

Fig. 5. Covariance function.

- Diffusion of gas in the liquid phase (Fick's law).

Liquid flow:

- Liquid phase (Darcy's law).

Mechanical behaviour:

- The behaviour of soil is dependent on stresses and suction.

The simulation of all these interacting phenomena requires the simultaneous solution of the equations of gas and liquid mass balance and linear momentum balance (equilibrium) together with the appropriate equilibrium restrictions and constitutive equations. An outline of the basic formulation is given below.

The formulation uses a multi-phase multi-species approach. The following assumptions are taken into account in the formulation of the problem: dry gas is considered a single species and it is the main component of the gaseous phase. Henry's law is used to express equilibrium of dissolved air. In a general nonisothermal formulation, thermal equilibrium between phases is assumed. Vapour concentration is in equilibrium with the liquid phase (the psychrometric law expresses its concentration).

State variables (also called unknowns) are: solid displacements, $\boldsymbol{u}$ (three spatial directions); liquid pressure, $p_{1}$; gas pressure, $p_{\mathrm{g}}$; and temperature, $T$. Balance of momentum for the medium as a whole is reduced to the equation of stress equilibrium together with a mechanical constitutive model to relate stresses with strains. Strains are defined in terms of displacements. Small strains and small strain rates are assumed for solid deformation. Advective terms due to solid displacement are neglected once the formulation is transformed in terms of material derivatives (in fact, material derivatives are approximated as eulerian time derivatives). In this way, volumetric strain is properly considered.

Balances of momentum for dissolved species and for fluid phases are reduced to constitutive equations (Fick's law and Darcy's law). Physical parameters in constitutive laws are, in general, function of pressure and temperature.

\subsection{Balance equations}

A summary of the balance equations are compiled here (further details are given in Olivella et al., 1994). The following notation is used: $\phi$ : porosity; $b$ : body forces; $\rho$ : density; $\omega$ : mass fraction; $\boldsymbol{j}$ : total mass flux; $\theta$ : mass content per unit volume of phase, i.e., $\theta=\omega$ $\rho$; $\boldsymbol{i}$ : non-advective mass flux; $E$ : specific internal energy; $q$ : advective flux; $\boldsymbol{i}_{\mathrm{c}}$ : conductive heat flux; $u$ : solid displacements; $\boldsymbol{j}_{\mathrm{E}}$ : energy fluxes due to mass motion; $\sigma$ : stress tensor; $S_{1}, S_{\mathrm{g}}$ : degree of saturation of liquid and gaseous phases, i.e., fraction of pore volume occupied by each phase. Superscripts w and a refer to water and dry gas, respectively. Subscripts s,

Table 3

Parameters of the random permeability fields

\begin{tabular}{llllll}
\hline Field name & Mean & Variance $(\log K)$ & Type of field & Range $D_{x}$ & Range $D_{z}$ \\
\hline 4 & $4.6 \times 10^{-19} \mathrm{~m}^{2}$ & 2 & SPHERIC & $1 \mathrm{~mm}=D_{x} / 50$ & $1 \mathrm{~mm}=D_{z} / 50$ \\
$4 \mathrm{a}$ & $4.6 \times 10^{-19} \mathrm{~m}^{2}$ & 2 & ANISOTROPIC & $1 \mathrm{~mm}=D_{x} / 50$ & $18 \mathrm{~mm}=9 / 25 D_{z}$ \\
$4 \mathrm{~b}$ & $4.6 \times 10^{-19} \mathrm{~m}^{2}$ & 2 & ANISOTROPIC & $1 \mathrm{~mm}=D_{x} / 50$ & $36 \mathrm{~mm}=18 / 25 D_{z}$ \\
\hline
\end{tabular}


1 and $g$ refer to solid, liquid and gas phase, respectively.

Water mass balance equation:

$\frac{\partial}{\partial t}$ (mass of water in liquid and gas phase)

+ divergence(total fluxes of water)

$=$ (external supply of water $)$

$\frac{\partial}{\partial t}\left(\theta_{1}^{\mathrm{w}} S_{\mathrm{l}} \phi+\theta_{\mathrm{g}}^{\mathrm{w}} S_{\mathrm{g}} \phi\right)+\nabla \cdot\left(j_{1}^{\mathrm{w}}+j_{\mathrm{g}}^{\mathrm{w}}\right)=f^{\mathrm{w}}$.

Dry gas mass balance equation:

$\frac{\partial}{\partial t}$ (mass of dry gas in liquid and gas phase)

+ divergence(total fluxes of dry gas)

$=($ external supply of dry gas $)$

$\frac{\partial}{\partial t}\left(\theta_{1}^{\mathrm{a}} S_{1} \phi+\theta_{\mathrm{g}}^{\mathrm{a}} S_{\mathrm{g}} \phi\right)+\nabla \cdot\left(\boldsymbol{j}_{1}^{\mathrm{a}}+\boldsymbol{j}_{\mathrm{g}}^{\mathrm{a}}\right)=f^{\mathrm{a}}$.

Internal energy balance equation:

$\frac{\partial}{\partial t}$ (internal energy in solid, liquid and gas phase)

+ divergence(total fluxes of energy)

$=$ (external supply of heat)

$\frac{\partial}{\partial t}\left(E_{\mathrm{s}} \rho_{\mathrm{s}}(1-\phi)+E_{\mathrm{l}} \rho_{1} S_{\mathrm{l}} \phi+E_{\mathrm{g}} \rho_{\mathrm{g}} S_{\mathrm{g}} \phi\right)$

$+\nabla \cdot\left(\boldsymbol{i}_{\mathrm{c}}+\boldsymbol{j}_{\mathrm{Es}}+\boldsymbol{j}_{\mathrm{El}}+\boldsymbol{j}_{\mathrm{Eg}}\right)=\mathrm{f}^{\mathrm{Q}}$.

\subsection{Constitutive equations and equilibrium restric-} tions

The formulation requires a set of necessary constitutive and equilibrium laws. Table 1 is a summary of the constitutive laws and equilibrium restrictions that have been incorporated to the model. The dependent variables, which are computed using each of the laws mentioned, are also included.

Additional relationships, which link dependent variables with unknowns are the equilibrium restrictions. They are obtained by assuming chemical equilibrium for dissolution of the different species (dry gas and vapour) in phases (liquid, gas). This assumption is sufficiently adequate because these chemical processes are fast compared to the transport processes

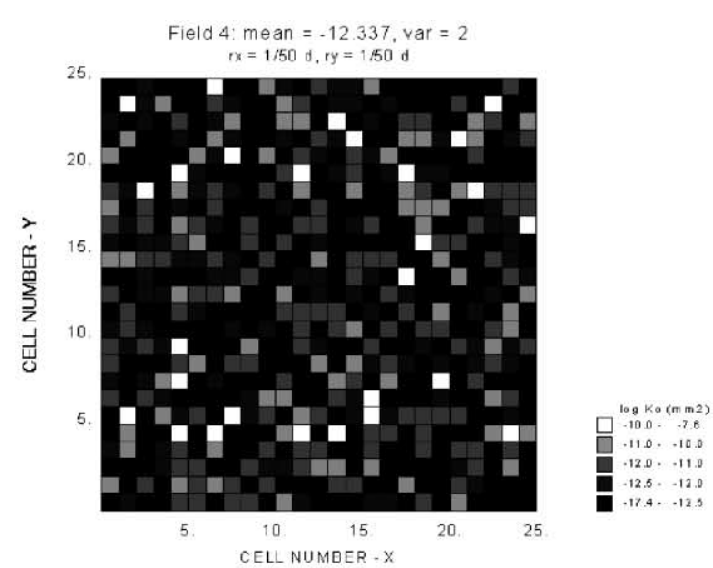

a) Field 4

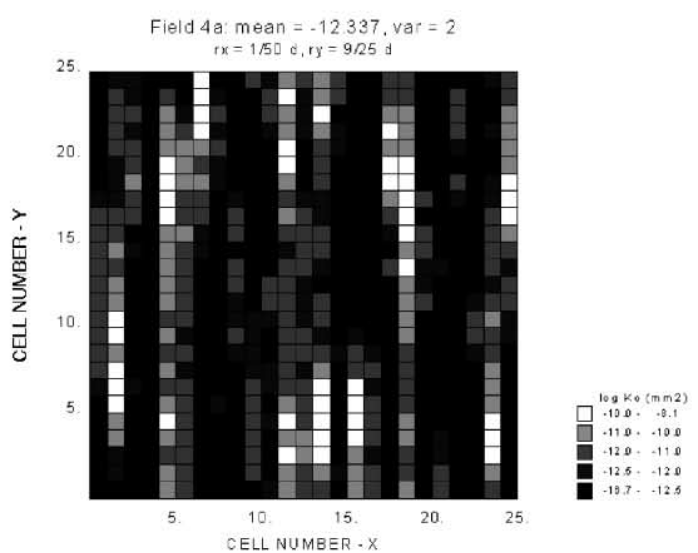

b) Field 4a

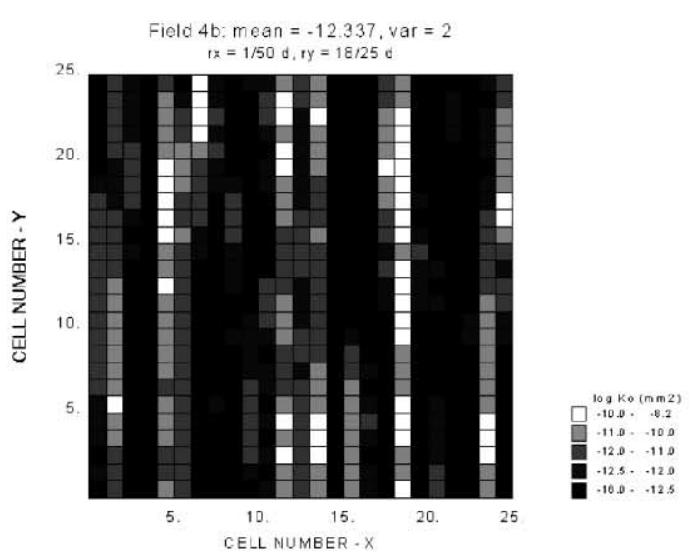

c) Field 4b

Fig. 6. Three realizations of random permeability fields. 


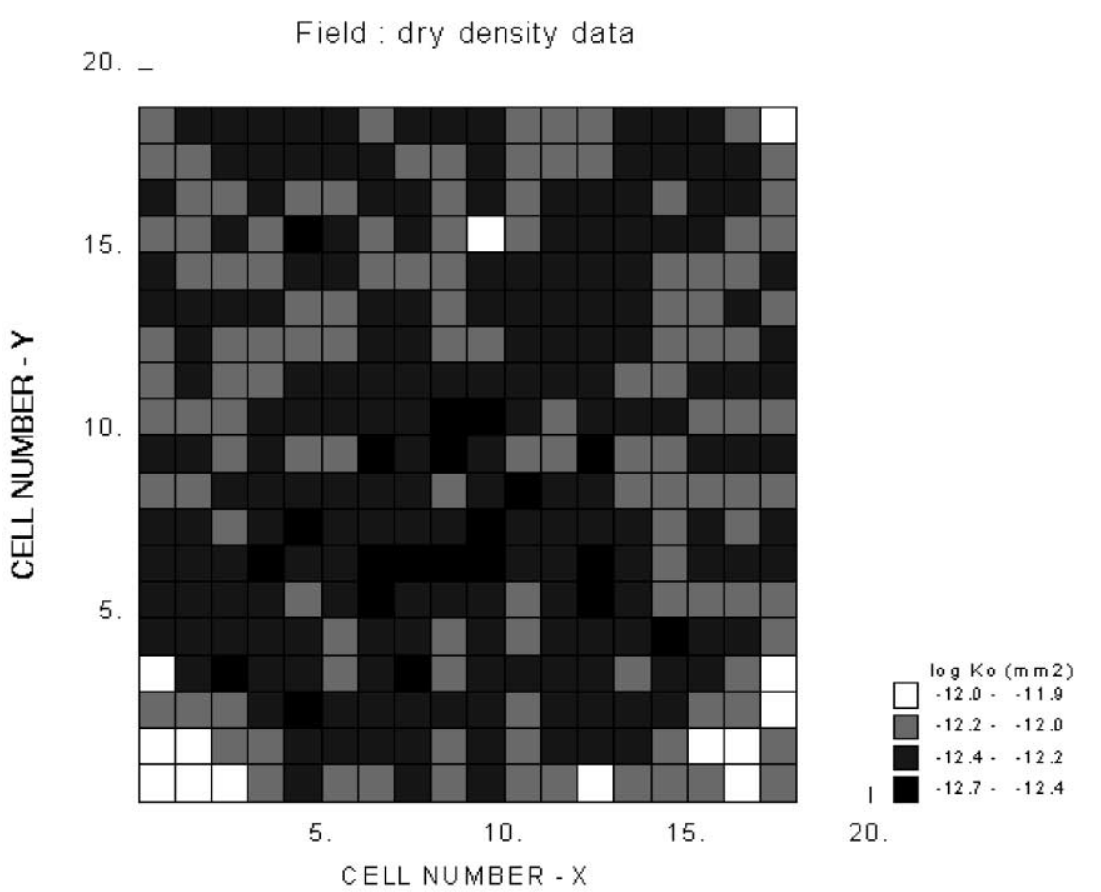

Fig. 7. Field of intrinsic permeability calculated from dry density data reported by Conciani et al. (1995).

that take place in porous media and, for this reason, they are not rate controlled.

\subsection{Boundary conditions}

Application of the Green's theorem to the divergence term (both in the balance and the stresses equilibrium equations) produces terms which represent fluxes or stresses across or on the boundaries. These terms are substituted by nodal flow rates or forces in the discretized form of the equations. For the mechanical problem, the classical approach is followed to impose external forces. Imposing displacement is made by means of a Cauchy type boundary condition, i.e., a force computed as the stiffness of a spring times the displacement increment. The boundary conditions for balance equations are incorporated by means of the simple addition of nodal flow rates. For instance, the mass flow rate of water as a component of gas phase (i.e., vapour) is:

$$
\begin{aligned}
j_{\mathrm{g}}^{\mathrm{w}}= & \left(\omega_{\mathrm{g}}^{\mathrm{w}}\right)^{0} j_{\mathrm{g}}^{0}+\left(\omega_{\mathrm{g}}^{\mathrm{w}}\right)^{0} \gamma_{\mathrm{g}}\left(P_{\mathrm{g}}^{0}-P_{\mathrm{g}}\right)+\beta_{\mathrm{g}}\left(\left(\rho_{\mathrm{g}} \omega_{\mathrm{g}}^{\mathrm{w}}\right)^{0}\right. \\
& \left.-\left(\rho_{\mathrm{g}} \omega_{\mathrm{g}}^{\mathrm{w}}\right)\right)
\end{aligned}
$$

where the superscript ()$^{0}$ stands for prescribed values. This general form of boundary condition includes three terms. The first one is the mass inflow or outflow that takes place when a flow rate of gas $\left(j_{\mathrm{g}}^{0}\right)$ is prescribed. The second term is the mass inflow or outflow that takes place when gas phase pressure $\left(P_{\mathrm{g}}^{0}\right)$ is prescribed at a node. The coefficient $\gamma_{\mathrm{g}}$ is a leakage coefficient, i.e., a parameter, which allows the specification of a boundary condition of the Cauchy type. The third term is the mass inflow or outflow that takes place when vapour mass fraction is prescribed at the boundary. This term naturally comes from the non-advective flux (Fick's law). Mass fraction and density prescribed values are only required when inflow takes place. For outflow, the values in the granular medium are considered. For the energy balance equation, the boundary condition has a similar form.

\subsection{Equilibrium restrictions}

Equilibrium restrictions are given for the concentration of dissolved dry gas in water. 


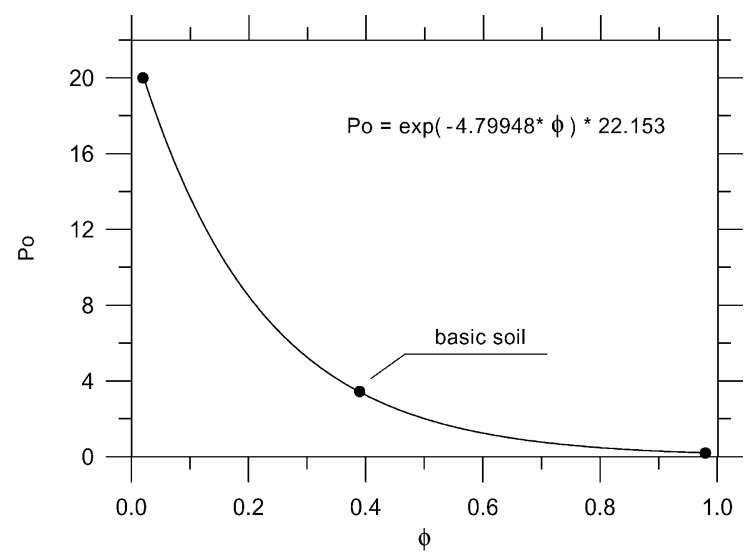

Fig. 8. Variation of $P_{0}$ parameter with soil porosity.

Solubility of dry gas in water is controlled by Henry's law:

$\theta_{1}^{\mathrm{a}}=\frac{P_{\mathrm{a}}}{H} \frac{M_{\mathrm{a}}}{M_{\mathrm{w}}} \rho_{1}$

where $\theta_{1}^{\mathrm{a}}$ is the mass fraction of dry gas per unit volume of liquid, $P_{\mathrm{a}}$ is the partial pressure of dry gas, $H$ is Henry's constant, $M_{\mathrm{a}}$ is the molecular mass of dry gas and $M_{\mathrm{w}}$ is the molecular mass of water. $\rho_{1}$ is the liquid density.

\subsection{Constitutive equations}

\subsubsection{Hydraulic}

Liquid and gas flows follow Darcy's law:

$\boldsymbol{q}_{1}=-\boldsymbol{K}_{1}\left(\nabla P_{1}-\rho_{1} \boldsymbol{g}\right)$

$\boldsymbol{q}_{\mathrm{g}}=-\boldsymbol{K}_{\mathrm{g}}\left(\nabla P_{\mathrm{g}}-\rho_{\mathrm{g}} \boldsymbol{g}\right)$

where $P_{1}$ and $P_{\mathrm{g}}$ are liquid and gas pressures, respectively, $\rho_{1}=\rho_{10} \exp \left(\beta\left(P_{1}-P_{10}\right)\right)(\beta=$ liquid compressibility) is the liquid density, $\rho_{\mathrm{g}}$ is the gas density and $\boldsymbol{K}_{\alpha}=\boldsymbol{k} k_{r \alpha} / \mu_{\alpha}$ is the permeability tensor. The intrinsic permeability tensor $(\boldsymbol{k})$ depends on the pore structure of the porous medium. $k_{r \alpha}$ is the value of relative permeability, which controls the variation of permeability in the unsaturated regime and $\mu \alpha$ denotes the dynamic viscosity. In this expressions, $\alpha$ may stand for either 1 or $g$ depending on whether liquid or gas flow is considered. The relative permeability of liquid and gaseous phases is made dependent on degree of saturation using empirical relationships. $g$ is the gravity vector.

The variation of intrinsic permeability with porosity is given by:

$k=k_{0} \frac{\phi^{3}}{(1-\phi)^{2}} \frac{\left(1-\phi_{0}\right)^{2}}{\phi_{0}^{3}}$

Table 4

Mechanical properties of Boom Clay, elastoplastic model

\begin{tabular}{lll}
\hline Property & Symbol & Numerical value \\
\hline Elastic & & $2.65 \times 10^{-2}$ \\
Elastic volumetric compressibility (against mean stress changes) & $k_{\mathrm{i} 0}$ & $3.22 \times 10^{-3}$ \\
Volumetric compressibility against suction changes & $k_{\mathrm{s} 0}$ & 0.333 \\
Poisson's ratio & $v$ & 0.26 \\
Plastic & & $2(0)$ \\
Elastoplastic volumetric compressibility & $r$ & 0.564 \\
Parameters to define LC yield curve & $\beta$ & $54.4 \mathrm{MPa}^{-1}$ \\
& $k$ & 0.1 \\
Parameters to define increase in strength with suction & $P_{\mathrm{c}}$ & $0.06 \mathrm{MPa}$ \\
Reference stress & $M$ & 1 \\
Slope of critical state & $\alpha$ & 0.395 \\
Parameter for the plastic potential & & $6 \mathrm{MPa}$ \\
Initial State & $p_{0}^{*}$ & 0.5881 \\
Initial preconsolidation stress for saturated conditions & $e_{0}$ & \\
Initial void ratio & &
\end{tabular}

Parameters not mentioned in the Table were set to zero. 
where $k_{0}$ is the intrinsic permeability corresponding to $\varphi_{0}$ (a reference porosity).

The relative permeability of liquid $\left(k_{\mathrm{rl}}\right)$ and gaseous $\left(k_{\mathrm{rg}}\right)$ phases is made dependent on $S_{\mathrm{e}}$ (effective degree of saturation) according to:

$S_{\mathrm{e}}=\frac{S_{\mathrm{l}}-S_{\mathrm{lr}}}{S_{\mathrm{ls}}-S_{\mathrm{lr}}} \leq 1$

$k_{\mathrm{rl}}=S_{\mathrm{e}}^{1 / 2}\left(1-\left(1-S_{\mathrm{e}}^{1 / \lambda}\right)^{\lambda}\right)^{2} \quad($ Van Genuchten $)$

$k_{\mathrm{rl}}=A S_{\mathrm{e}}^{n} \quad($ Generalised Power law $)$

$k_{\mathrm{rg}}=A\left(1-S_{\mathrm{e}}\right)^{\beta} \quad($ Generalised Power law $)$

where $S_{1}$ is degree of saturation, $S_{\mathrm{lr}}$ and $S_{\mathrm{ls}}$ are residual and maximum degree of saturation, respectively, and $\lambda, n, A$ and $\beta$ are material parameters.

It is also necessary to define the retention curve of the materials by relating degree of saturation with suction $\left(s=\left(P_{\mathrm{g}}-P_{1}\right) \geq 0\right)$. The expression selected is (Van Genuchten, 1980):

$S_{\mathrm{e}}=\frac{S_{1}-S_{\mathrm{lr}}}{S_{\mathrm{ls}}-S_{\mathrm{lr}}}=\left(1+\left(\frac{s}{P_{0}}\right)^{1 / 1-\lambda}\right)^{-\lambda}$

where $P_{0}$ is a parameter related to the capillary pressure and $\lambda$ is a parameter which controls the shape of the curve. Hysteresis effects have not been taken into account in the analysis.

Finally, molecular diffusion of dry gas in liquid is governed by Fick's law:

$\boldsymbol{i}_{1}^{\mathrm{a}}=-\boldsymbol{D}_{1}^{\mathrm{a}} \nabla \omega_{1}^{\mathrm{a}}=-\left(\phi \rho_{1} S_{1} \tau D_{\mathrm{m}}^{\mathrm{a}} \boldsymbol{I}+\rho_{\mathrm{l}} \boldsymbol{D}_{1}^{\prime}\right) \nabla \omega_{1}^{\mathrm{a}}$

where $\boldsymbol{i}_{1}^{\mathrm{a}}$ is the non-advective mass flux of gas in liquid, $\boldsymbol{D}_{1}^{\mathrm{a}}$ is the dispersion tensor, $\omega_{1}^{\mathrm{a}}$ is the mass fraction of gas in liquid, $\tau$ is the tortuosity and $\boldsymbol{D}_{1}^{\prime}$ the mechanical dispersion tensor. $D_{\mathrm{m}}^{\mathrm{a}}$ is the molecular diffusion coefficient of dry gas in liquid.

\subsubsection{Mechanical}

In saturated porous materials, mechanical behaviour is best understood in terms of effective stress: $\sigma^{\prime}=\sigma-P_{1} \boldsymbol{m}$, where $\boldsymbol{m}^{T}$ is the auxiliary vector

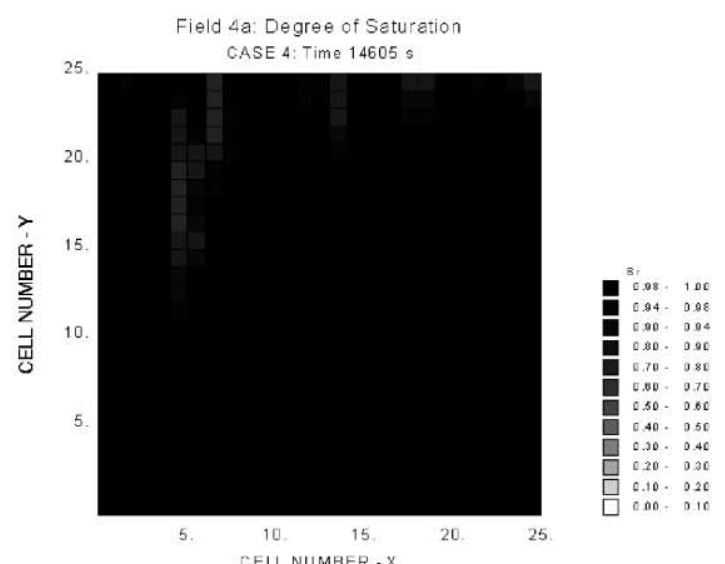

a) Time $=14605 \mathrm{~s}$

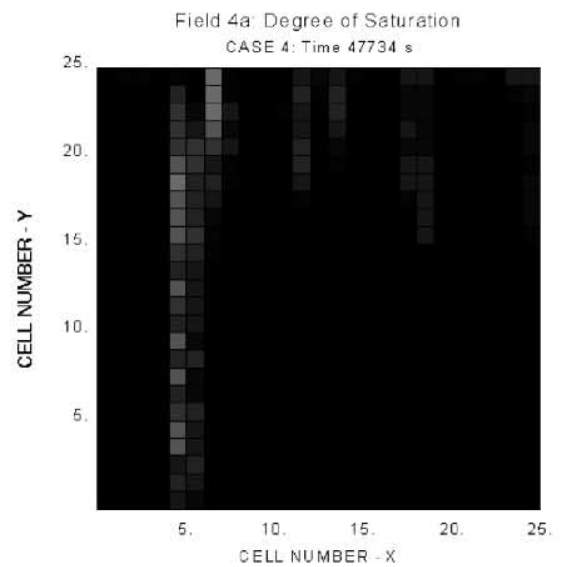

b) Time $=47734 \mathrm{~s}$

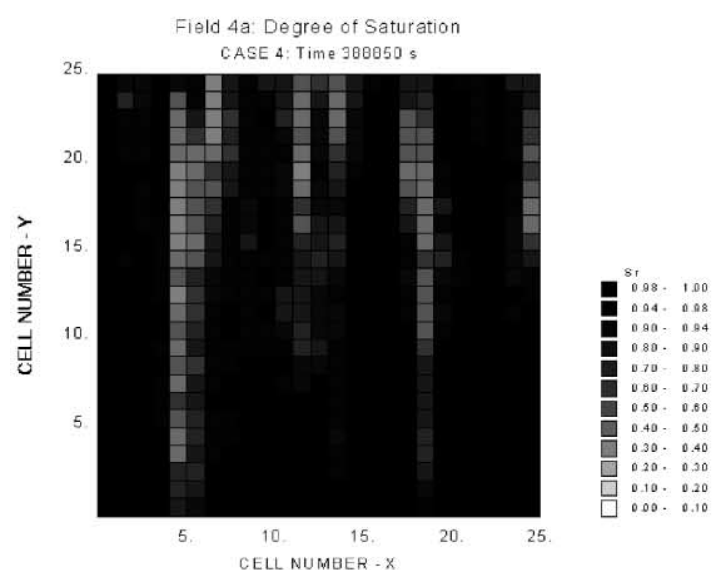

c) Time $=\mathbf{3 8 8 8 5 0 ~} \mathrm{s}$

Fig. 9. Preferential path formation in Case 4, Field 4a. 

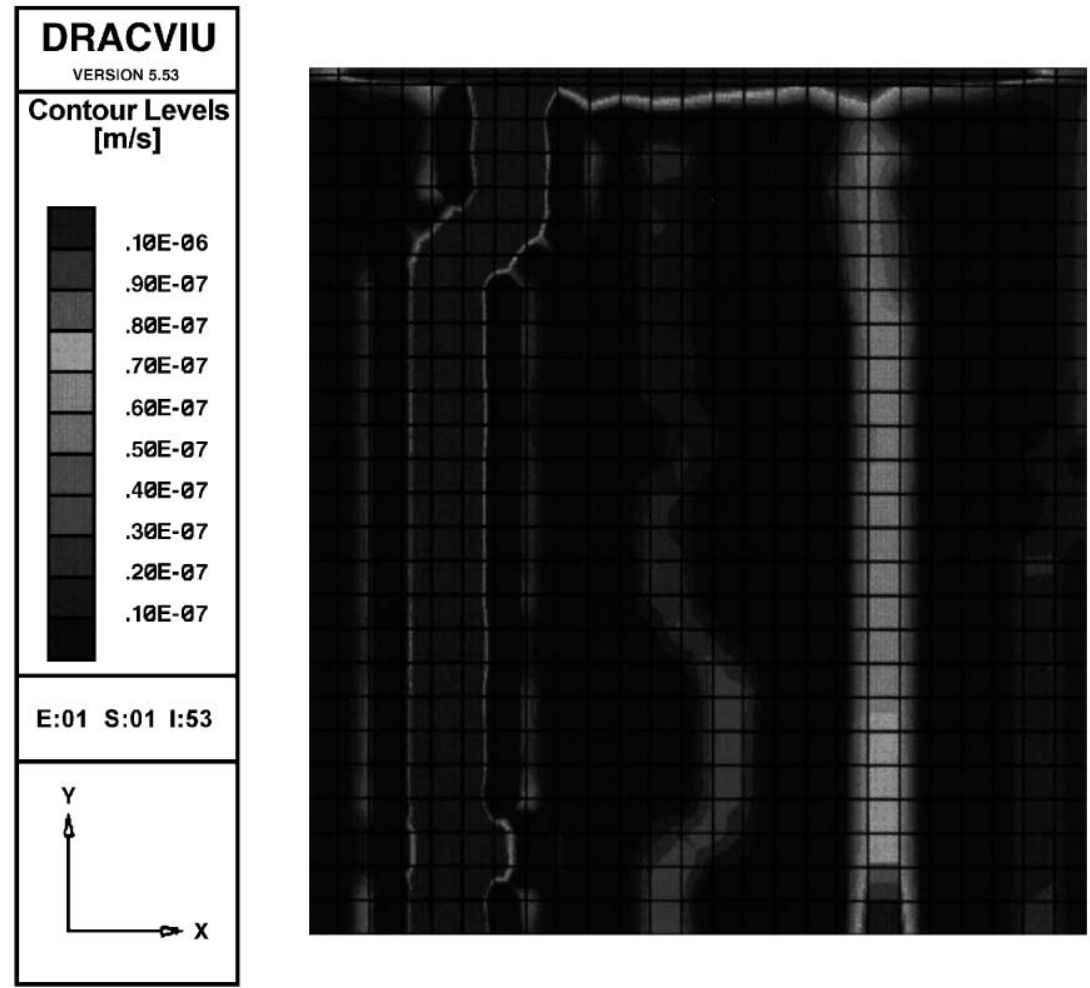

Fig. 10. Case 4. Field 4a. Gas velocity contours.

$[1,1,1,0,0,0]$. For unsaturated materials, it is necessary to consider two independent stress variables (Gens, 1995a). In this work, net stresses $\left(\sigma-P_{\mathrm{g}} \boldsymbol{m}\right)$ and capillary suction, $s=\left(P_{\mathrm{g}}-P_{1}\right)$ have been adopted as independent stress variables. Net stress is the excess of total stress over gas pressure. If full saturation is achieved, net mean stress becomes effective stress. For simplicity, net stress will also be denoted by $\sigma$.

The mechanical constitutive equation takes, in general, an incremental form:

$\mathrm{d} \sigma=\boldsymbol{D} \mathrm{d} \varepsilon+\boldsymbol{h} \mathrm{d} s+\beta \mathrm{d} T$.

An elastoplastic constitutive law (Alonso et al., 1990; Gens, 1995b) has been selected to perform the analyses reported later. In the remaining of the paper, isothermal conditions will be assumed.

In terms of stress invariants, the isothermal yield surface may be written as:

$f=f\left(p, J, \theta, \varepsilon_{\mathrm{v}}^{0}, s\right)$ where:

$$
\begin{aligned}
& p=\left(\frac{1}{3}\right)\left(\sigma_{x}+\sigma_{y}+\sigma_{z}\right) \\
& J^{2}=1 / 2 \operatorname{trace}\left(s^{2}\right) \\
& \theta=-\frac{1}{3} \sin ^{-1}\left(1.5 \sqrt{3} \operatorname{det} s / J^{3}\right) \\
& s=\sigma-p \boldsymbol{I}
\end{aligned}
$$

$\boldsymbol{I}$ is the identity tensor. For simplicity, a form of the classical Modified Cam-clay model is taken as the reference isothermal saturated constitutive law:

$F=\frac{3 J^{2}}{g_{y}^{2}}-L_{y}^{2}\left(p+P_{\mathrm{s}}\right)\left(P_{0}-p\right)=0$.

Volumetric strain is defined as:

$\varepsilon_{\mathrm{v}}=\varepsilon_{x}+\varepsilon_{y}+\varepsilon_{z}$

and $\varepsilon_{\mathrm{v}}^{\mathrm{p}}$ is the plastic volumetric strain. 


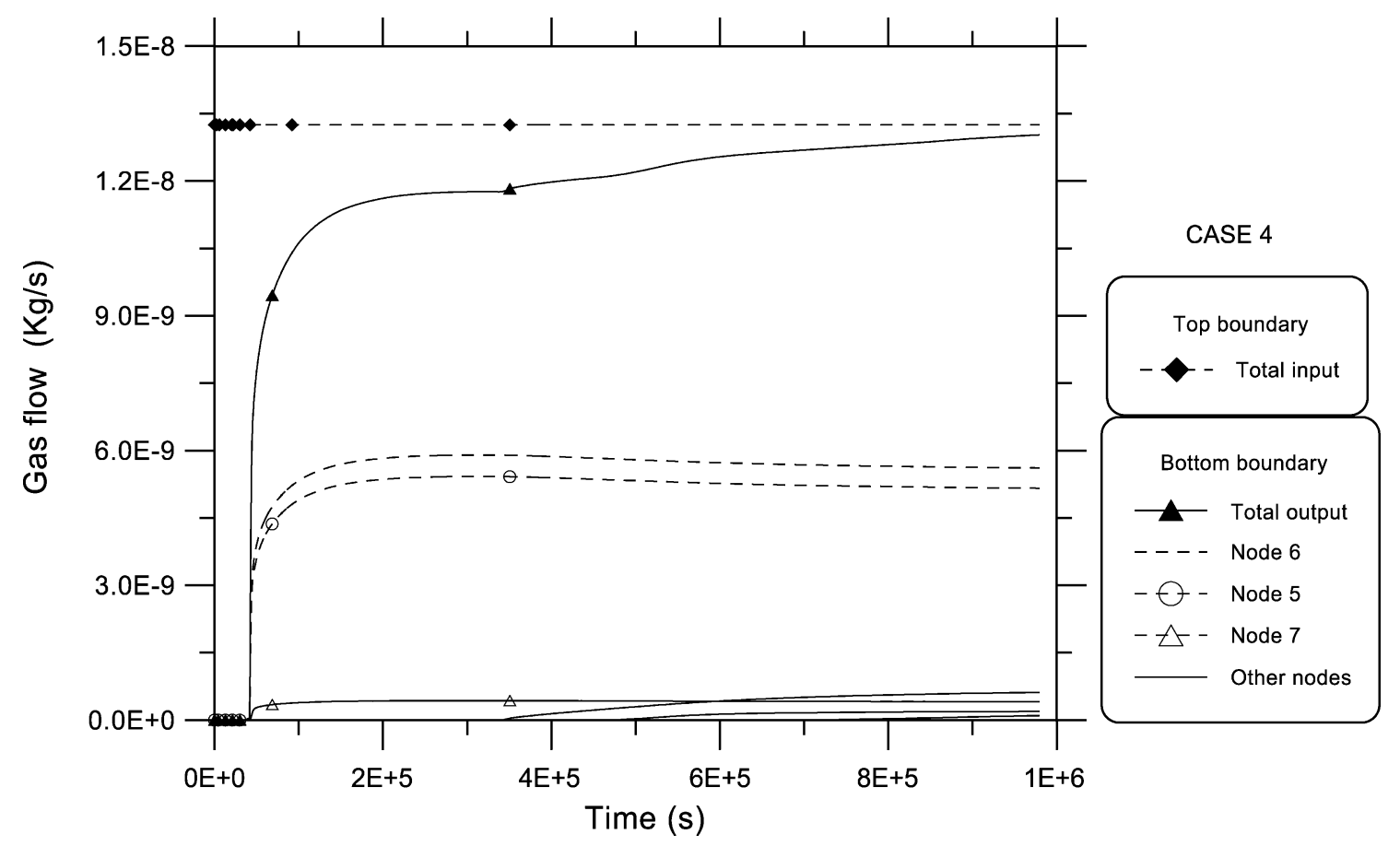

Fig. 11. Case 4. Computed gas flow output. Field 4a.

The basic assumption is that the preconsolidation pressure, $P_{0}$, depends on suction:

$P_{0}=P_{\mathrm{c}}\left(\frac{P_{0}^{*}}{P_{\mathrm{c}}}\right)^{\frac{\lambda(0)-k_{\mathrm{i} 0}}{\lambda(\mathrm{s})-k_{\mathrm{i} 0}}}$

where $P_{0}^{*}$ is the saturated preconsolidation mean stress and $P_{\mathrm{c}}$ is a reference stress. $\lambda(\mathrm{s})$ is the soil compressibility coefficient, which is made dependent on suction. $k_{\mathrm{i} 0}$ is an elasticity coefficient defined below.

$L_{y}=\frac{M}{g_{y}(\theta=-\pi / 6)}$

where $M$ is the slope of the critical state failure line and $g_{y}$ is a function of Lode angle (DIT-UPC, 2000).

$\lambda(\mathrm{s})=\lambda(0)[(1-r) \exp (-\beta s)+r]$

where $r$ and $\beta$ are material constants.

$P_{\mathrm{s}}$ expresses the increase in apparent cohesion with suction:

$P_{\mathrm{s}}=k s$

where $k$ is a material constant.
It was assumed that hardening depends on plastic volumetric strain only according to:

$\dot{P}_{0}^{*}=\frac{1+e}{\lambda(0)-k_{\mathrm{i} 0}} P_{0}^{*} \dot{\varepsilon}_{\mathrm{v}}^{\mathrm{p}}$

and the plastic potential reads:

$G=\frac{3 J^{2}}{g_{\mathrm{p}}^{2}}-\alpha L_{\mathrm{p}}^{2}\left(p+P_{\mathrm{s}}\right)\left(P_{0}-p\right)$.

If $\alpha=1$, an associated plastic model results.

Elastic stiffness changes with suction. The following simple model was adopted:

$\dot{\varepsilon}_{\mathrm{v}}^{\mathrm{e}}=\frac{k_{\mathrm{i}}}{1+e} \frac{\dot{P}}{P}+\frac{k_{\mathrm{s}}}{1+e} \frac{\dot{s}}{s+0.1}$

$k_{\mathrm{i}}=k_{\mathrm{i} 0}\left(1+\alpha_{\mathrm{is}} s\right)$

$k_{\mathrm{s}}=k_{\mathrm{s} 0}\left(1+\alpha_{\mathrm{sp}} \ln p / P_{\mathrm{r}}\right) \exp \left(\alpha_{\mathrm{ss}} s\right)$

$\dot{E}=\dot{J} / G ; \quad G=E / 2(1+v)$ 
where $k_{\mathrm{i} 0}, k_{\mathrm{s} 0}, \alpha_{\mathrm{is}}, \alpha_{\mathrm{sp}}, \alpha_{\mathrm{ss}}, P_{\mathrm{r}}$ are material parameters, $E$ is the deviatoric strain and $E, v$ the classical elasticity constants.

The preceding set of equations describes the model used in simulations. The meaning of the soil material parameters is discussed in Alonso et al. (1990) and DIT-UPC (2000). Further details will be given in the next section.

\section{Simulation of soil heterogeneity}

\subsection{Reference homogeneous soil}

Basic soil properties of Boom Clay, as described in Delahaye and Alonso (1997), have been selected as the reference common set of parameters for the analysis presented here. The water retention curve for the homogeneous case (basic soil) is given in Fig. 1 for the porosity $n=0.39$. It is the best approximation to actual laboratory data. The relative permeabilities of gas and water follow the interpolation curves indicated in Fig. 2. The boundary value problem solved is indicated in Fig. 3. It approaches the one-dimensional gas flow experiments performed by Harrington and Horseman (1997) on samples of Boom Clay. A constant gas flow rate is imposed against one of the sample ends. On the opposite face water pressure is maintained constant $\left(p_{\mathrm{w}}=2.21\right.$ $\mathrm{MPa}$ ). The lateral surface is impervious to both gas and water. The sample is confined under an isotropic stress $\sigma=4.4 \mathrm{MPa}$ and it is initially saturated, the pore water pressure being $p_{\mathrm{w}}=2.21 \mathrm{MPa}$. Six computer models of this basic test (Cases 1 to 6) have been developed (see Table 2). Case 1 corresponds to the homogeneous sample just described. It is a purely two-phase flow problem without any mechanical interaction.

Simulations for Case 1 and indeed for the remaining cases have been performed under 2D, plane strain conditions in order to keep the problem tractable from a numerical point of view. In fact, nonlinearities of the numerical problem, specially in cases of full hydro-mechanical interaction, make the problem very demanding in terms of computer power. It was decided to maintain all the simulations reported here within the framework of a $2 \mathrm{D}$ geometry.

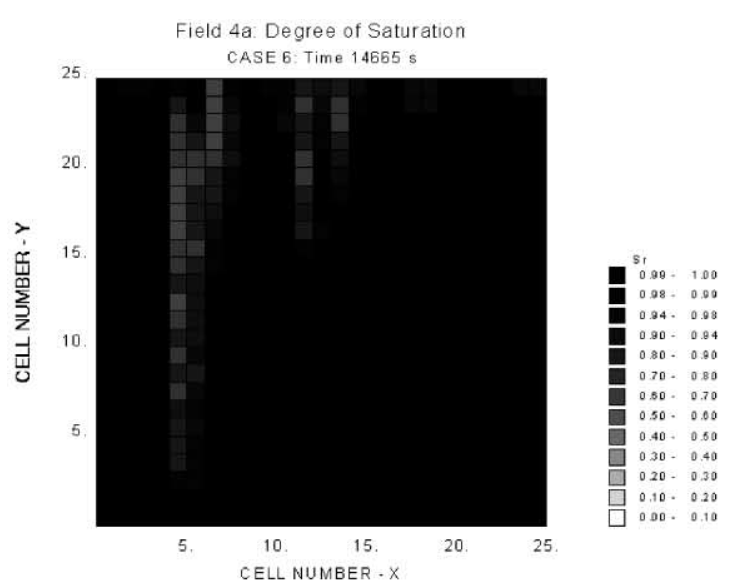

a) Time $=14665 \mathrm{~s}$

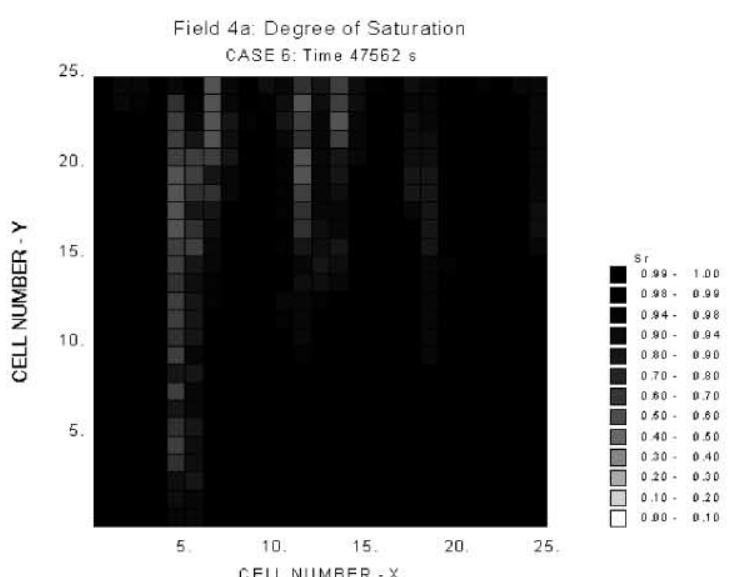

b) Time $=\mathbf{4 7 5 6 2 ~ s}$

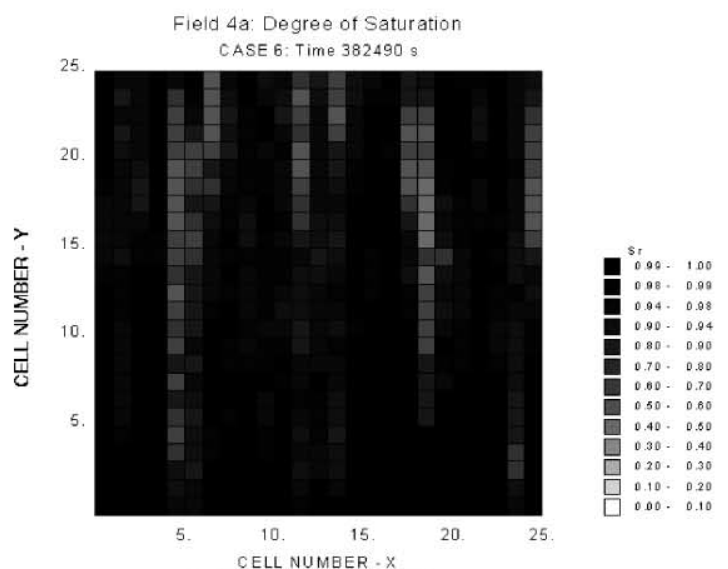

c) Time $=382490 \mathrm{~s}$

Fig. 12. Preferential path formation in Case 6. 


\subsection{Heterogeneous soil}

As described in the Introduction, the purpose of the paper is to explore the conditions for the development of preferential paths in heterogeneous soils. The idea is schematically indicated in Fig. 4. In a three-dimensional case (Fig. 4a), a preferential path will be defined as a juxtaposition of pervious spots. In this paper, the problem has been simplified to two dimensions (plane strain conditions for the coupled hydromechanical analysis) as sketched in Fig. 4b.

\subsubsection{Random permeability fields}

Permeability was simulated as a two-dimensional stationary random permeability field, with a constant mean and a covariance function that depends only on the relative distance vector between two arbitrary points. Covariance function is defined by its variance and its range.

Both magnitudes are shown in Fig. 5 for a onedimensional random field $Z(x)$. The underlying probability density function of the permeability field $K(z, x)$ was described by a log normal distribution. An anisotropic random field distribution was considered in order to simulate preferred orientations within the soil. A typical feature of a natural soil leading to a marked anisotropy in soil permeability is its layered structure, resulting from a sedimentary deposition. Two correlation ranges have been considered: $d_{x}$ along $x$ direction and $d_{z}$ along the $z$ direction. When the correlation range increases, the spatial variability is reduced. A zero correlation distance leads to the maximum randomness ("white noise").

Most of the analyses performed correspond to a reference permeability field whose characteristics are indicated in Table 3 (Field 4a). Its mean value corresponds to the reference intrinsic permeability adopted for the homogeneous reference case $(4.6 \times$ $10^{-19} \mathrm{~m}^{2}$ ).

The variance of $\log K$ (a normal random field, since $K$ has a log-normal distribution) is relatively large (Var $[\log K]=2)$ and implies significant changes in permeability over short distances. Anisotropy is defined by two different ranges along the $x$ and $z$ directions: $d_{x}=1$

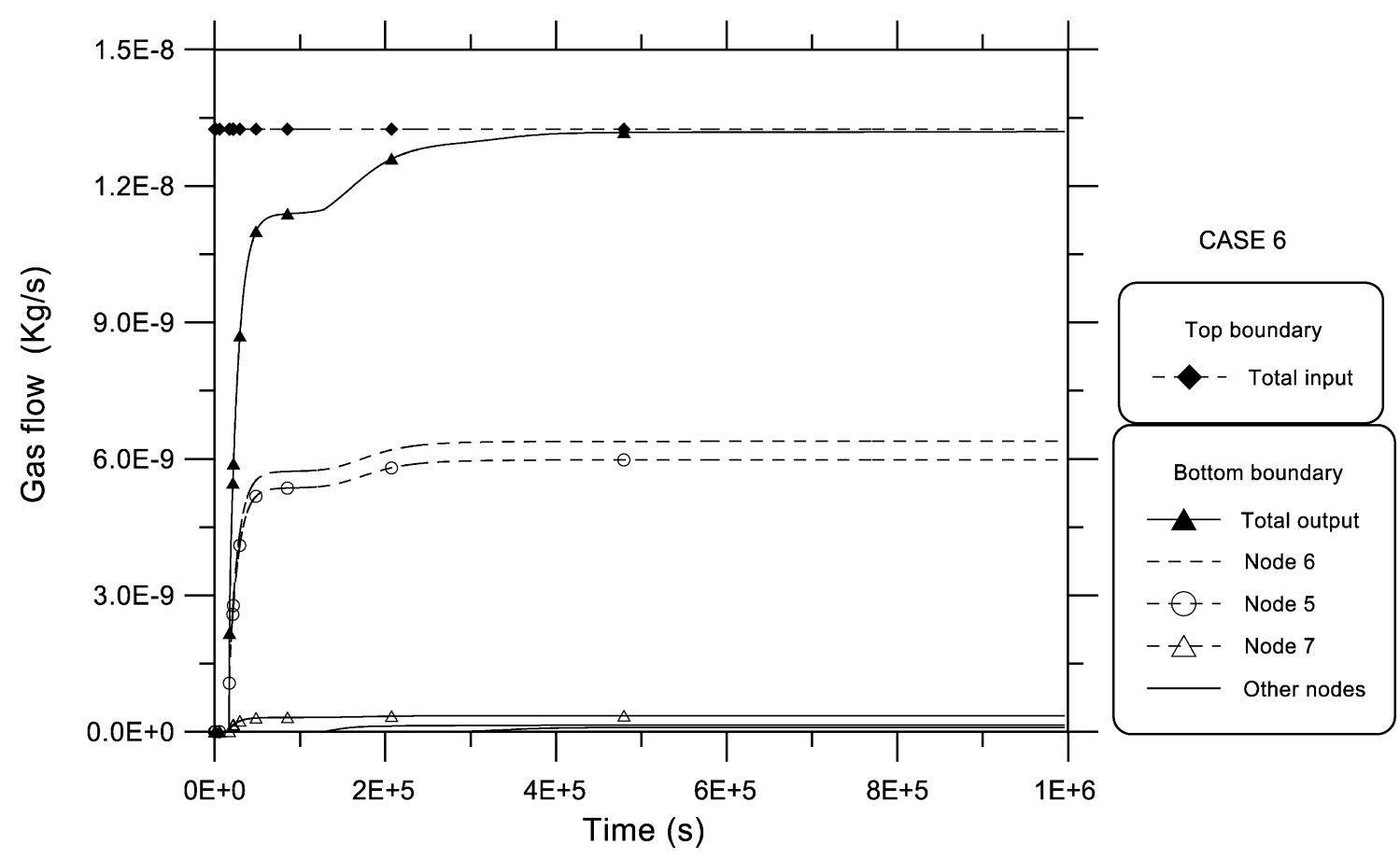

Fig. 13. Case 6. Computed gas flow output. 


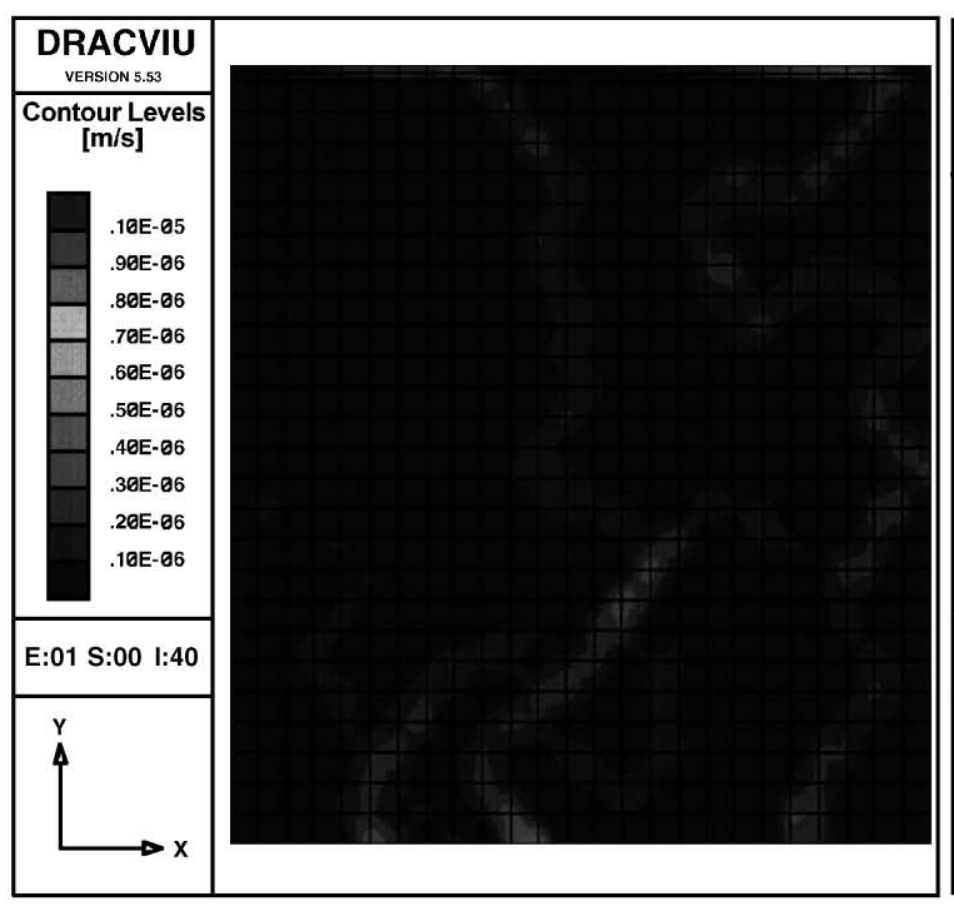

(a)

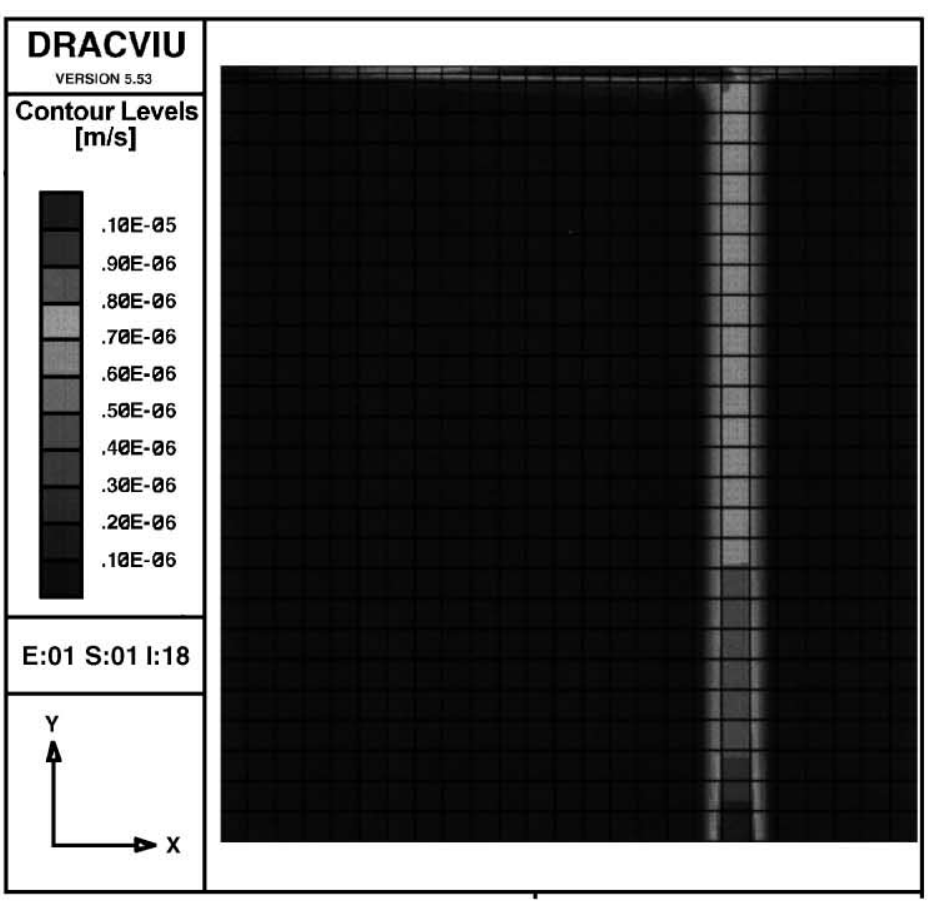

(b)

Fig. 14. Gas velocity contours for Case 4. (a) Random permeability Field 4 (isotropic); (b) random permeability Field 4b (highly anisotropic). 
$\mathrm{mm}=D_{x} / 50$ and $d_{z}=18 \mathrm{~mm}=9 / 25 D_{z}$, where $D_{x}$ and $D_{z}$ are the width and height of the two-dimensional sample $\left(D_{x}=D_{z}=50 \mathrm{~mm}\right.$, see Fig. 3). Two additional random fields were also considered: Field 4 (a spherical random field having a very small correlation distance) and Field $4 \mathrm{~b}$ (an anisotropic field whose correlation range along the $z$ direction has been increased with respect to Case $4 a$, see Table 3 ). The $5 \times 5 \mathrm{~cm}$ "sample" was divided, in all cases, in $25 \times 25$ equal square cells $(2 \times 2 \mathrm{~mm})$. Each one of these cells was modelled by means of a finite element with constant properties. Fig. 6 shows three realisations of permeability distributions for the three random fields defined above. The effect of different correlation distances is clearly shown in Fig. 6.

Some micrographic studies of soil variability have revealed that natural soils may exhibit significant changes in properties over short distances. This is indicated in Fig. 7, which represents the variation of soil intrinsic permeability calculated from dry density data reported by Conciani et al. (1995) for a Brazilian soil. The technique used gave in this case information on $2 \times 2 \mathrm{~mm}$ areas. Porosity changed in the range
$0.31-0.49$. Porosity changes alone do not give enough information to derive permeability, which may be much controlled also by changes in soil composition, mineralogy and microstructure. These results provide, however, a clue on the scale of fluctuation that which may be present in natural soils.

\subsubsection{Hydraulic analysis. Cases defined}

Cases 2, 3 and 4 are defined in Table 2. Since changes in permeability are likely to be associated with simultaneous changes in porosity and water retention properties, an approximate procedure to extend the generated permeability field to changes in porosity and retention characteristics has been introduced. Porosity is approximated by back-calculation through the wellknown Kozeny's relationship. Then a set of water retention curves of varying gas entry value as porosity changes was adopted as shown in Fig. 1. The relationship between porosity and the $P_{0}$ parameter of Van Genuchten law is shown in Fig. 8. The rest of parameters of the reference retention curve did not change.

Case 4 represents the full hydraulic variability: Every cell is in itself a different "soil" characterised

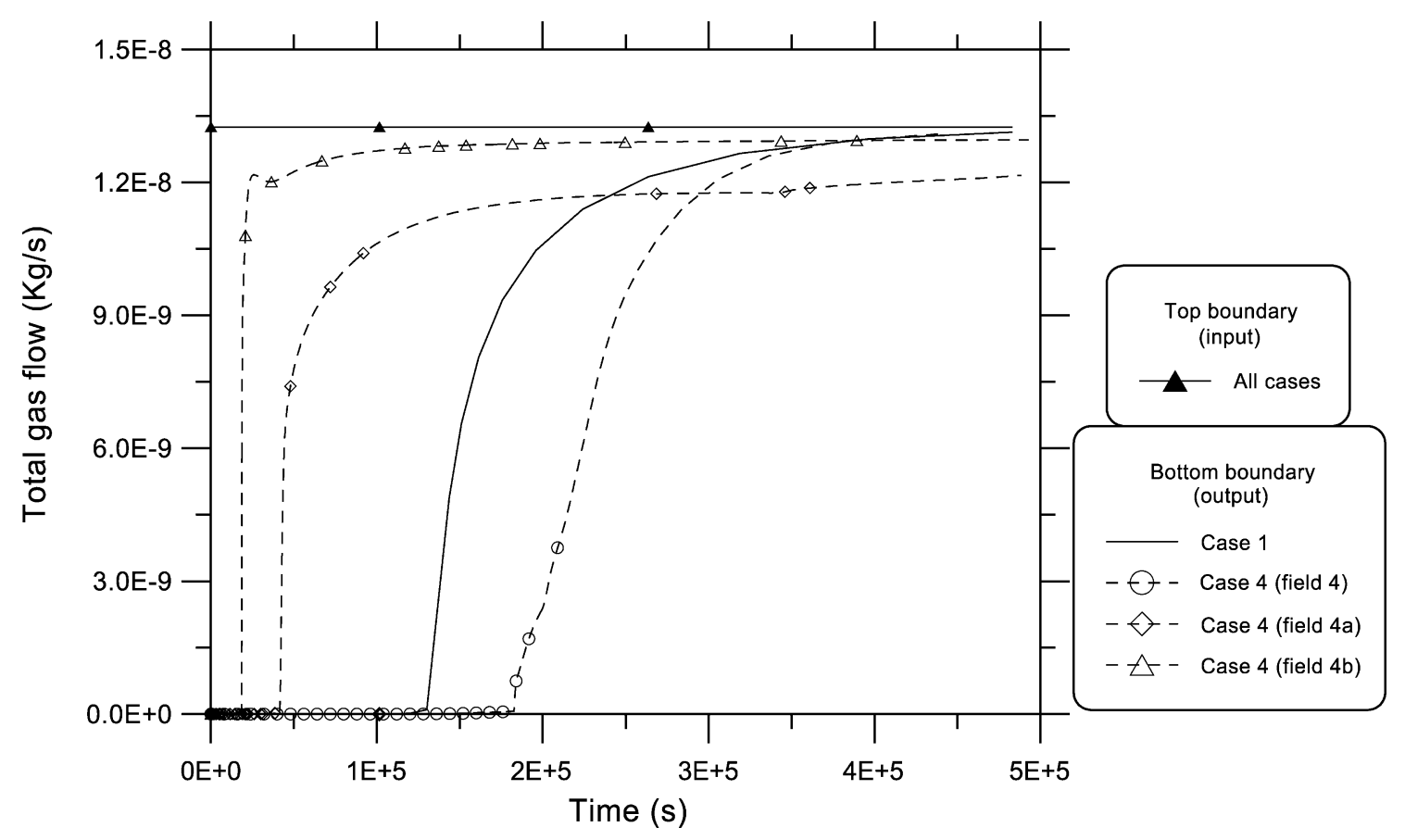

Fig. 15. Case 4. Effect of spatial structure on soil variability. Computed gas outflow. 

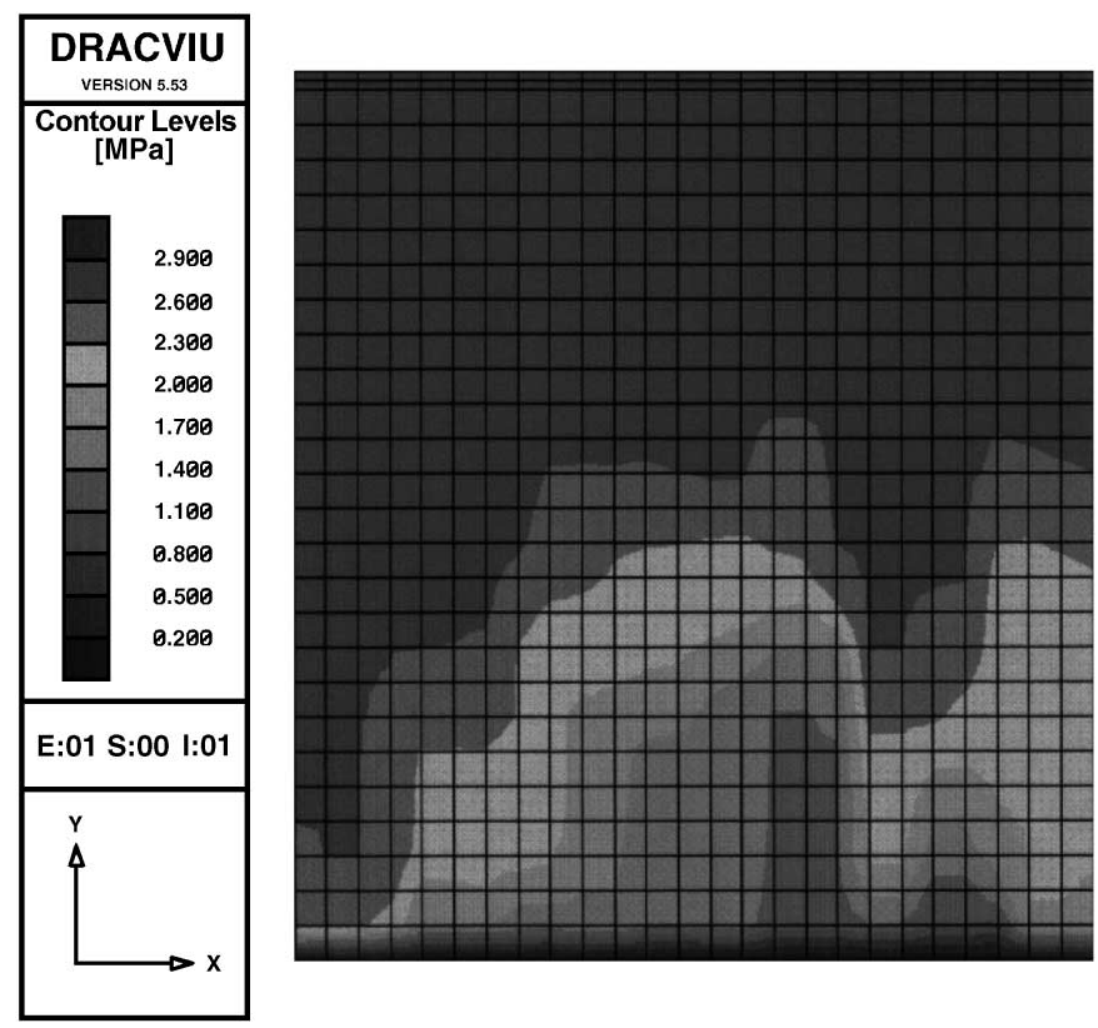

Fig. 16. Suction contours for Case 5, permeability Field 4a at steady state.

by its own porosity, intrinsic permeability and water retention characteristics. Relative permeabilities were kept unchanged, however. Cases 2 and 3 are simpler versions of Case 4 as may be seen in Table 2 .

A "consistency" condition has been introduced to all the defined cases in order to preserve a common average hydraulic conductivity. As the sample becomes heterogeneous (Cases 2, 3, 4) preferential paths lead to a higher average conductivity. In order to maintain the hydraulic conductivity of the soil sample equal to the original value $\left(K_{0}=4.6 \times 10^{-19} \mathrm{~m}^{2}\right)$, the average intrinsic permeability, $K_{0}$, was reduced for Cases 2 to 6 . This is done through a simulated "permeability test" under saturated conditions. The following intrinsic average permeabilities were found:

$$
\begin{array}{ll}
\text { Case 2, 5: } & K_{0}=7.5 \times 10^{-20} \mathrm{~m}^{2} \\
\text { Case 3, 4, 6: } & K_{0}=8.18 \times 10^{-21} \mathrm{~m}^{2} .
\end{array}
$$

\subsubsection{Coupled flow-mechanical analysis. Cases defined}

Cases 1 to 4 have a rigid soil skeleton. In the last two cases defined (5 and 6), the soil is assumed to deform through the appropriate constitutive law, which has been defined in terms of two stress fields, net stress and suction, as described previously. One consequence of the hydro-mechanical coupling is that as the gas pressure increases, the net stress (defined as the excess of total stress over gas pressure) decreases and an expansion of the soil will ensue. This in turn will increase the permeability.

In Case 5 (Table 2), a simple linear elastic relationship with parameters $E=75 \mathrm{MPa}, v=0.333$ was adopted. Otherwise the hydraulic framework is equal to Case 3. Finally, in Case 6, the hydraulic framework adopted is equal to Case 4 and a comprehensive elastoplastic model was introduced. The fundamentals of this model were given before. The set of material mechan- 
ical parameters for Case 6 is indicated in Table 4. They were taken from DIT-UPC (1997).

\section{Results}

Only two cases will be discussed here in some detail to avoid a lengthy presentation: Case 4 , which includes the maximum hydraulic complexity, and Case 6, which introduces a mechanical coupling.

\subsection{Case 4 (defined in Table 2)}

The process of gas invasion of the initially saturated sample may be followed in Fig. 9, which shows the degree of saturation computed at three time instants after the beginning of the test. The graphs show the progressive development of preferential paths, which become unsaturated. Eventually, at steady state conditions, a major path is established and the remaining area of the sample is crossed by less significant paths.
This is shown in Fig. 10, which provides the gas velocity contours at steady state (for Field 4a). The computed gas flow rate at the bottom of the sample is shown in Fig. 11. It shows the major contribution of two nodes (nodes 5 and 6 located at the lower left-hand side of the domain) and the small contributions of other nodes (associated with the secondary paths identified in Fig. 10). Also indicated is the total flow rate that gradually converges toward the total input gas flow rate.

\subsection{Case 6 (defined in Table 2)}

Similar plots to the previous case, but now introducing mechanical interaction, are given in Figs. 12 and 13. Fig. 12 gives an indication of the development of preferential paths for times similar to the times selected for Case 4 (and Field 4a). The mechanical effect is visible: it enhances the development of preferential paths as the gas pressure decreases the net stresses within the sample.
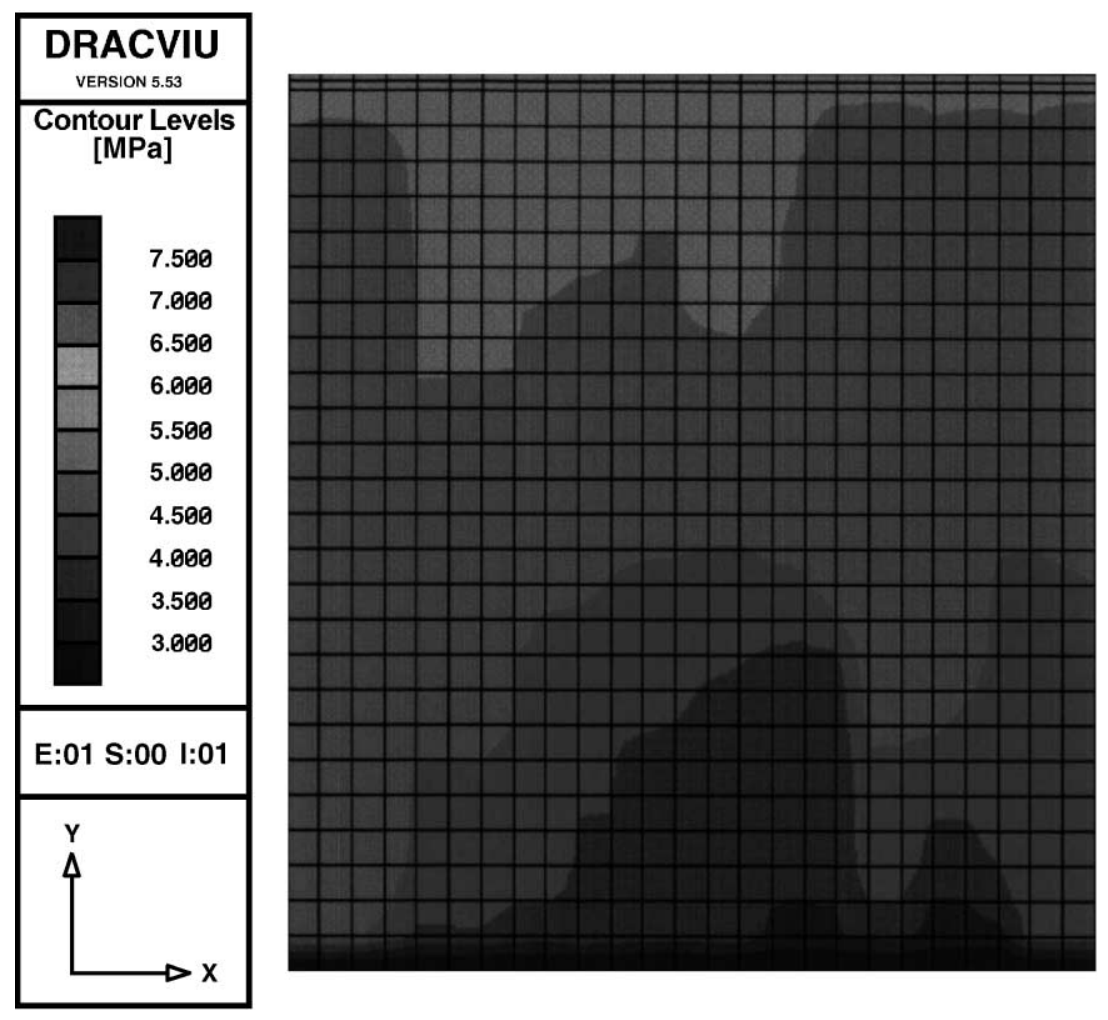

Fig. 17. Gas pressure field for Case 5, permeability Field 4a at steady state. 
A larger number of significant preferential paths are computed now. The time history of output gas flow rate shows (Fig. 13) an earlier time to breakthrough that is a consistent result in view of the previous comments.

\section{The effect of changing soil heterogeneity}

The spatial structure of soil variability has a profound effect on computed results. This comment is illustrated in Fig. 14a and b, which give a graphical representation of preferential paths for Case 4 and the two extreme random permeability fields defined in Table 3 (Fields 4 and $4 b$ ).

For the small scale of fluctuation and isotropic case (Field 4), a tortuous path scheme is found. Several paths eventually daylight at the bottom end. Fields $4 \mathrm{a}$ and $4 \mathrm{~b}$, characterised by a strong anisotropy along the imposed average gas flow, result in a progressively more defined, straight passage of flow. The time history of output gas flow rate reflects these features (Fig. 15). In fact, the strongest the anisotropy in correlation, the earlier the time for breakthrough, which may change in a very significant way.

\section{Discussion}

A few additional features of the computed results and a comparison of cases will be presented here. Fig.
16 shows a suction contour plot for Case 5, permeability field $4 \mathrm{a}$. The irregularities demonstrate the soil heterogeneity and the presence of preferential paths. However, there is a consistent desaturation pattern along the sample. The variation of average suction (or degree of saturation) along the flow path will indicate a fairly continuous variation from a dry end, close to the gas injection boundary, to a wet end on the opposite side. This is a result actually measured in tests, which was also discussed in a previous paper (Delahaye and Alonso, 1997).

The gas pressure field for the same case (Fig. 17) reflects also the soil heterogeneity. Spots with higher gas pressure will have a reduced net stress and therefore an increased porosity (and permeability). The development of preferential paths is thus enhanced.

A comparison of all cases analysed is presented in Figs. 18, 19 and 20 in terms of time histories of: total gas flow rate, total liquid flow rate (computed at the bottom end of the samples) and differential pressure $\left(p_{\mathrm{g}}-p_{\mathrm{w}}\right)$ between the upper and lower ends of the sample.

Fig. 18 indicates that the introduction of a spatial random variation of hydraulic properties results in a reduction of time to breakthrough and, in general, a different time history of gas flow rate. The reference case is the homogeneous sample (Case 1). As discussed before, this is a consequence of the natural development of preferential paths. The consideration of a mechanical coupling (Cases 5 and 6) provides an

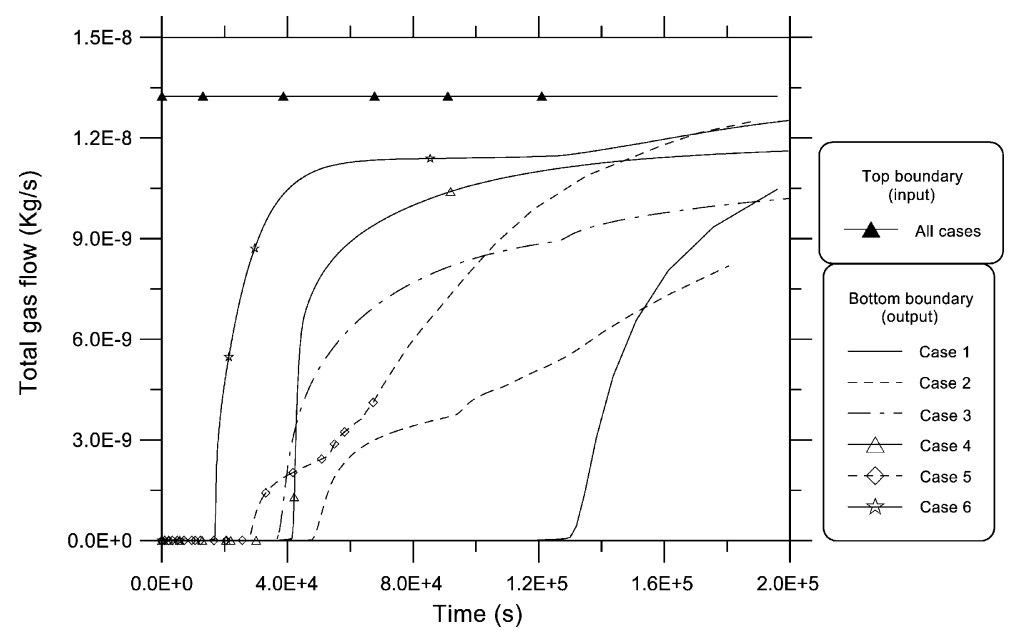

Fig. 18. Total gas flow output rate. Comparison of Cases 1 through 6. 


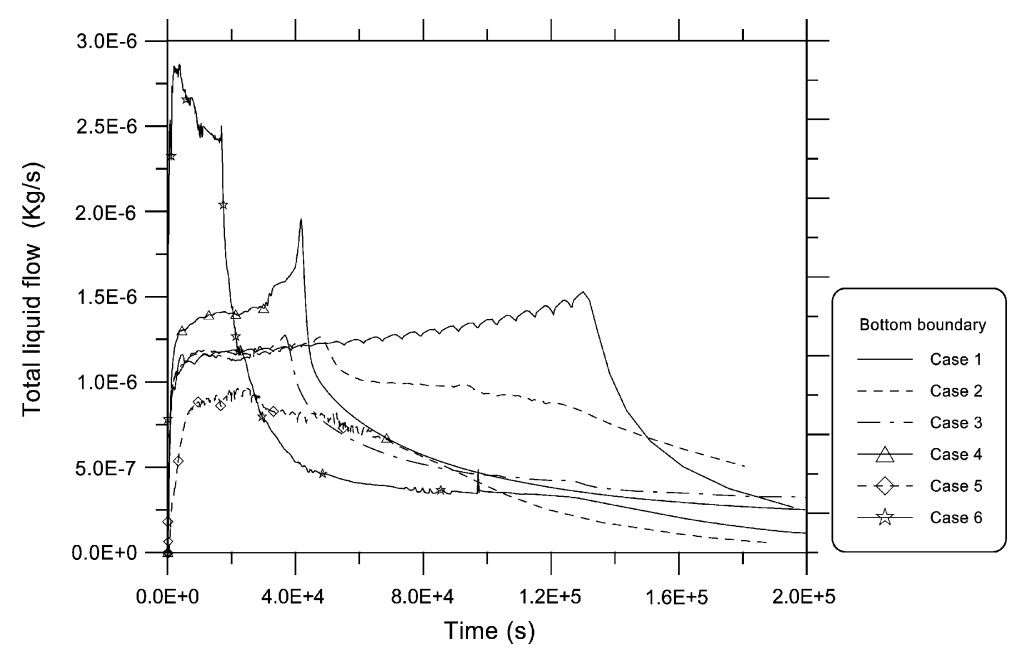

Fig. 19. Total liquid flow output rate. Comparison of Cases 1 through 6.

additional reduction of breakthrough time and a faster output gas flow rate thereafter (Fig. 21).

The total liquid output (Fig. 19) shows a similar pattern for all the cases analysed. Liquid flow rate increases up to a maximum and then decreases steadily. If Figs. 18 and 19 are compared, the peak in liquid flow rate is shown to coincide approximately with the time for gas breakthrough. The release of gas through the lower end implies also a progressive reduction of gas flow. The computed differential pressure (Fig. 20) varies with the different hypothesis concerning the description of soil heterogeneity and mechanical coupling. Case 4, which may be considered a closer approximation to the actual soil variability, exhibits a distinct peak of differential pressure, which is close to the time for breakthrough. Cases 1 to 3 (purely hydraulic) also show a maximum, whereas in the coupled analysis (Cases 5 to 6), a steady increase in differential pressure is computed. It is suspected that the differential pressure history is sensitive to some parameters, which have been left unchanged during the analysis performed. For instance, the residual satura-

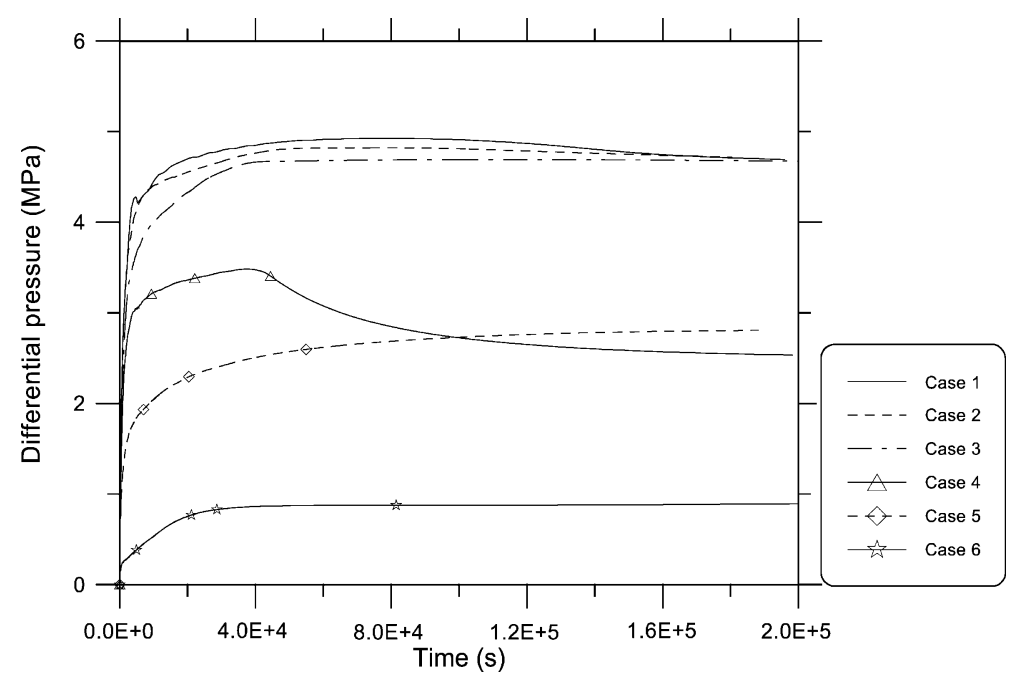

Fig. 20. Time history of differential pressure. Comparison of Cases 1 through 6 . 


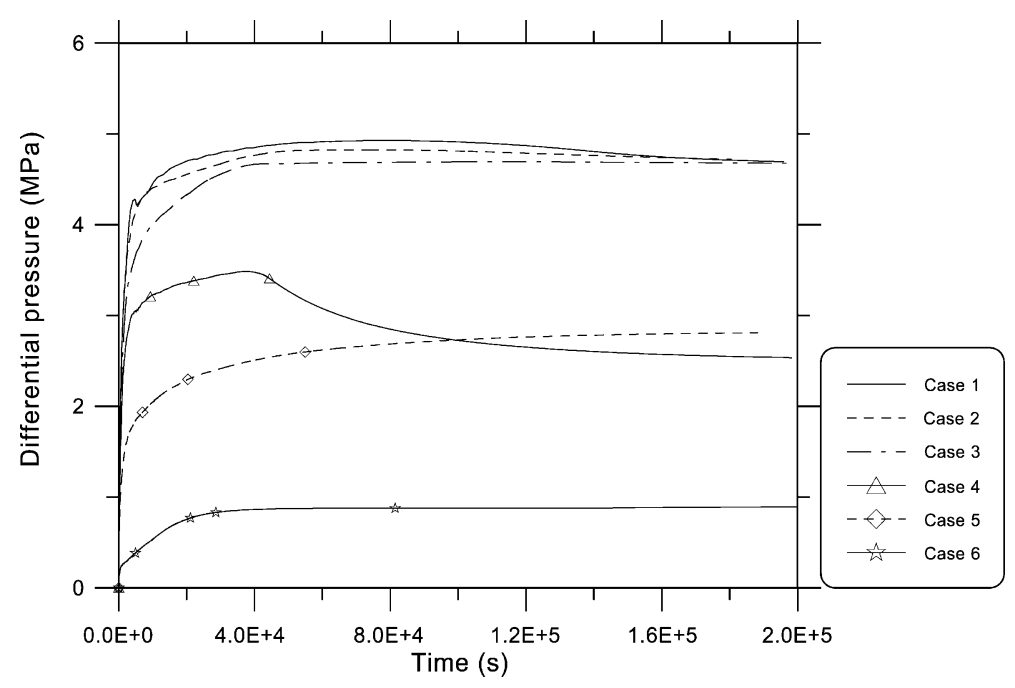

Fig. 21. Time history of differential pressure. Comparison of Cases 1 through 6.

tion value (a parameter of the water retention relationship) may contribute to the development of this peak.

\section{Conclusions}

This paper has explored the effects of natural soil variability on gas migration. The results presented indicate that the development of preferential paths may be a natural consequence of soil heterogeneity. The accurate description of soil heterogeneity is hampered by the lack of reliable data and therefore, a sensitivity analysis has been carried out. The analysis is obviously not complete since the amount of significant variables that may control the process is exceedingly large. An additional important consideration is the large computational effort that is required to perform a comprehensive analysis from a probabilistic point of view. The results presented correspond to a simple realisation of the random fields defined. The next step would be the performance of a Monte Carlo simulation in order to derive the statistics of the significant features of the problem (time to gas breakthrough, maximum differential pressure, etc). Nevertheless, the analysis carried out has demonstrated a number of interesting features.

- The spatial structure of natural fluctuations controls the breakthrough time. The smaller the correlation distance, the larger the breakthrough time.
- Consideration of the soil stiffness decreases the breakthrough time and increases the output gas flow rate. This is a consequence of the increased capability of the gas pressure to open preferential paths, a feature which is conveniently handled by the mechanical model, formulated in terms of net stress and suction.

- Water output flow rates have a maximum when the breakthrough time for gas is reached. Afterwards, water flow rates decrease steadily.

- A peak differential pressure between gas and water pressure at both ends of the sample is predicted by the hydraulic models used. It is likely that this behaviour is linked to some specific soil properties.

The emphasis in this paper is to demonstrate the effects of soil heterogeneity. No specific attempts have been made to reproduce soil tests, although average properties were approximated on the basis of known properties for Boom Clay. Major uncertainties remain in the quantification of variability. The analysis can be improved at the cost of a very large computational effort by introducing three-dimensional effects and full Monte Carlo simulations.

\section{Acknowledgements}

The research presented in this paper was financed through the EC PROGRESS project (FI4W-CT960024). 


\section{References}

Alonso, E.E., 1997. Flow and hydraulic fracture in earthfill dams. Commission Internationale des Grands Barrages. 19th Congress, Florence, Q73 R34, 521-549.

Alonso, E.E., Gens, A., Josa, A., 1990. A constitutive model for partially saturated soils. Géotechnique 40 (3), 405-430.

Aylmore, L.A.G., 1993. Use of computer assisted tomography in studying water movement around plant roots. Advances in Agronomy $49,1-53$.

Brun, P., 1989. Cinétique d'infiltration au sein d'une conche d'argile compactée. Etude expérimentale et numérique, Thèse, ENS Mines de Paris.

Conciani, W., Soares, M.M., Crestana, S., 1995. Geotechnical use of mini tomography. UNSAT'95. In: Alonso, E.E., Delage, P. (Eds.), Proc. First Int. Conf. On Unsat. Soils. Unsaturated Soils, vol. 2. Balkema.

Delahaye, C., Alonso, E.E., 1997. Modeling laboratory gas migration experiments. Project on Effects of Gas in Underground Storage Facilities for Radioactive Waste (Pegasus Project) Compiled by B. Haijtink and W. Rodwell. CEC. DOC XII/297/97EN.

DIT-UPC, 1997. Reseal Project: Scoping calculations. Internal Report, UPC, Spain.

DIT-UPC, 2000. CODE-BRIGHT. A 3-D program for thermohydro-mechanical analysis in geological media. User's guide. CIMNE, Barcelona.
Gens, A., 1995a. Constitutive modelling: application to compacted soils. In: Alonso, E.E., Delage, P. (Eds.), Unsaturated Soils, vol. 3. Balkema, Rotterdam, pp. 1179-1200.

Gens, A., 1995b. Constitutive laws. In: Gens, A., Jouanna, P., Schrefler, B.A. (Eds.), Modern Issues in Non-Saturated Soils. Springer Verlag, Wein, pp. 129-158.

Harrington, J.F., Horseman, S.T., 1997. Gas migration in clay. Project on Effects of Gas in Underground Storage Facilities for Radioactive Waste (Pegasus Project) CEC. DOC XII/297/97-EN.

Olivella, S., Carrera, J., Gens, A., Alonso, E.E., 1994. Nonisothermal multiphase flow of brine and gas through saline media. Transport in Porous Media 15, 271-293.

Olivella, S., Gens, A., Carrera, J., Alonso, E.E., 1996. Numerical formulation for a simulator (CODE-BRIGHT) for the coupled analysis of saline media. Engineering Computations 13, 87-112.

Ortiz, L., Volckaert, G., De Cannière, P., Put, M., Horseman, S.T., Harrington, J.F., Impey, M.D., Einchcomb, S.J.B., 1997. MEGAS: modelling and experiments on gas migration in repository host rocks. CEC. Final Report - Phase 2, EUR 17453EN.

Van Genuchten, M.T., 1980. A closed form equation for predicting the hydraulic conductivity of unsaturated soils. Soil Science Society of America Journal 44 (5), 892-898.

Volckaert, G., Ortiz, L., De Cannière, P., Put, M., Horseman, S.T., Harrington, J.F., Fioravante, V., Impey, M.D., 1995. MEGAS: modelling and experiments on gas migration in repository host rocks. CEC. Final Report-Phase 1, Eur 16235-EN. 